Int. J. Solids Structures, 40(6), 1505-1523 (2003)

\title{
On the Stress-Wave Imaging of Cavities in a Semi-Infinite Solid
}

\author{
Bojan B. Guzina ${ }^{\dagger}$, Sylvain Nintcheu Fata ${ }^{\dagger}$, and Marc Bonnet ${ }^{\ddagger}$ \\ ${ }^{\dagger}$ Dept. of Civil Engineering, University of Minnesota, Minneapolis, MN 55455 \\ ${ }_{\ddagger}^{\ddagger}$ Laboratoire de Mécanique des Solides, Ecole Polytechnique, F-91128 Palaiseau Cedex, France
}

\begin{abstract}
The problem of mapping underground cavities from surface seismic measurements is investigated within the framework of a regularized boundary integral equation (BIE) method. With the ground modeled as a uniform elastic half-space, the inverse analysis of elastic waves scattered by a threedimensional void is formulated as a task of minimizing the misfit between experimental observations and theoretical predictions for an assumed void geometry. For an accurate treatment of the gradient search technique employed to solve the inverse problem, sensitivities of the predictive BIE model with respect to cavity parameters are evaluated semi-analytically using an adjoint problem approach and a continuum kinematics description. Several key features of the formulation, including the rigorous treatment of the radiation condition for semi-infinite solids, modeling of an illuminating seismic wave field, and treatment of the prior information, are highlighted. A set of numerical examples with spherical and ellipsoidal cavity geometries is included to illustrate the performance of the method. It is shown that the featured adjoint problem approach reduces the computational requirements by an order of magnitude relative to conventional finite difference estimates, thus rendering the 3D elasticwave imaging of solids tractable for engineering applications.
\end{abstract}




\section{Introduction}

Remote sensing of cavities embedded in a semi-infinite solid via elastic waves is a topic of considerable interest in mechanics and engineering owing to its relevance to a number of applications ranging from nondestructive material testing to medical diagnosis, oil prospecting and underground object detection. In the context of seismic exploration, a comprehensive three-dimensional mapping of subterranean structures is typically associated with the interpretation of a large number (often thousands) of motion measurements via finite-difference elastodynamic models which are inherently based on domain discretization (e.g. Plessix et al., 1999). In contrast, this investigation is concerned with problems where detailed mapping of underground openings (such as defense facilities) is required and only a few measurements can be made, usually on the ground surface. In such instances, the boundary integral equation (BIE) formulations, which provide a direct mathematical link between the observed waveforms and the geometry of a hidden object, can be used to effectively compensate for the limited field data (see Colton and Kress, 1983, for acoustic problems).

The problem of inverse scattering (Bui, 1994), of interest in this study, has been the subject of extensive mathematical research; among numerous reviews on the topic, on may mention Colton and

Kress (1992), Colton et al. (2000), and Pike and Sabatier (2002) as examples spanning the past decade. In the context of impenetrable scatterers (such as voids examined herein), various numerical solution procedures, often based on the BIE method, have been proposed for the problem. Most existing treatments of this type, however, are limited to the inversion of electromagnetic or acoustic far-field waveforms in infinite media (e.g. Bonnet, 1995a). Few exceptions dealing with the inverse scattering of elastic waves include crack identification in infinite elastic solids by Kress (1996) (2D treatment in the frequency domain) and Nishimura (1997) (3D analysis in the time domain). Aimed at bridging such gap between the elastic wave scattering theory and its applications, the focus of this investigation is the development of an analytical and computational framework for the identification

of cavities via an elastodynamic BIE method, for the more complex and realistic case involving three-dimensional elastic wave propagation in a semi-infinite solid. By means of a well-defined incident seismic field and a set of surface motion sensors used to monitor elastic waves scattered by the cavity, the inverse problem is reduced to the minimization of a cost function representing the misfit between the field observations and their predictions for an assumed void location. For a precise treatment of the featured body and surface wave fields, the predictive model used in this study (Pak and Guzina, 1999) is based on the fundamental solution for a uniform elastic half-space.

In the pursuit of the gradient search technique employed by the inverse solution, necessary derivatives of the cost function are evaluated via an adjoint problem approach which, besides the matter of elegance, offers a superior computational performance relative to finite-difference sensitivity estimates. This is accomplished by revisiting the semi-analytical treatment proposed for 
infinite media in Bonnet (1995a). To establish a rigorous foundation for the imaging problem, included is an explicit treatment of the radiation condition for semi-infinite solids; a topic that has, despite its central role in the application of BIE methods to forward and inverse scattering problems involving unbounded media, eluded previous studies. A numerical example where an ellipsoidal cavity is identified from synthetically-generated, noise-polluted field measurements is included to illustrate the proposed method.

\section{Problem statement}

To establish a fundamental framework for the BIE-based identification of underground cavities by elastic waves, the focus of this study is the inverse scattering problem for an isotropic, homogeneous elastic half-space housing an internal void. With reference to a Cartesian frame $\left\{O ; \xi_{1}, \xi_{2}, \xi_{3}\right\}$, the half-space $\Omega=\left\{\left(\xi_{1}, \xi_{2}, \xi_{3}\right) \mid \xi_{3}>0\right\}$ is characterized by the Lamé's constants $\lambda$ and $\mu$, mass density $\rho$,

and is bounded on top by the free surface $S=\left\{\left(\xi_{1}, \xi_{2}, \xi_{3}\right) \mid \xi_{3}=0\right\}$. The cavity inside the half-space occupies a simply connected finite region $\Omega_{\mathrm{C}} \subset \Omega$ bounded by a smooth closed surface $\Gamma$. For further reference, let $\Omega^{-}$denote the semi-infinite region surrounding the cavity, i.e. the complement to $\Omega_{\mathrm{C}}$ in

the half-space so that $\Omega^{-}=\Omega \backslash\left(\Omega_{\mathrm{C}} \cup \Gamma\right)$, and let $\boldsymbol{n}$ denote the normal to $\Gamma$ directed towards the exterior of $\Omega^{-}$. With reference to Fig. 1 , the cavity is "illuminated" by a time-harmonic seismic source $\boldsymbol{f}$, with the resulting surface motion monitored over a finite set of slightly embedded control points $\boldsymbol{\xi}=\boldsymbol{x}^{m}(m=1,2, \ldots M)$. To discuss conditions at infinity, an auxiliary surface $\Gamma_{R}(\boldsymbol{\xi})=\Sigma(\boldsymbol{\xi}, R) \cap \Omega$ is introduced, where $\Sigma(\boldsymbol{\xi}, R)$ is the sphere of radius $R$ centered at $\boldsymbol{\xi} \in \Omega$. The respective subsets of $\Omega, \Omega^{-}$and $S$ which are bounded by $\Gamma_{R}$ will be denoted as $\Omega_{R}, \Omega_{R}^{-}$and $S_{R}$, with an implicit assumption that $R$ is sufficiently large so that $\Omega_{\mathrm{C}} \subset \Omega_{R}$.

\subsection{Forward problem}

For a systematic treatment of the inverse problem, it is necessary first to introduce the associated forward problem wherein the response of a semi-infinite solid $\Omega^{-}$due to prescribed seismic loading is to be determined for a known cavity location and geometry. With the time factor $e^{i \omega t}$ omitted henceforth for brevity, the forward solution, herein denoted as the total field $\boldsymbol{u}(\boldsymbol{\xi}, \omega)$, can be formally defined via an elastodynamic state $[\boldsymbol{u}, \boldsymbol{t}]$ which satisfies the field equations

$$
\begin{gathered}
\nabla \cdot \boldsymbol{\sigma}+\boldsymbol{f}=-\rho \omega^{2} \boldsymbol{u} \\
\quad \boldsymbol{\sigma}=\mathbf{C}: \boldsymbol{\varepsilon}, \quad \boldsymbol{\xi} \in \boldsymbol{\Omega}^{-} \\
\boldsymbol{\varepsilon}=\frac{1}{2}\left(\nabla \boldsymbol{u}+\nabla^{T} \boldsymbol{u}\right),
\end{gathered}
$$

subject to the boundary conditions

$$
\boldsymbol{t} \equiv \boldsymbol{\sigma} \cdot \boldsymbol{n}=\mathbf{0}, \quad \boldsymbol{\xi} \in \Gamma \cup S
$$




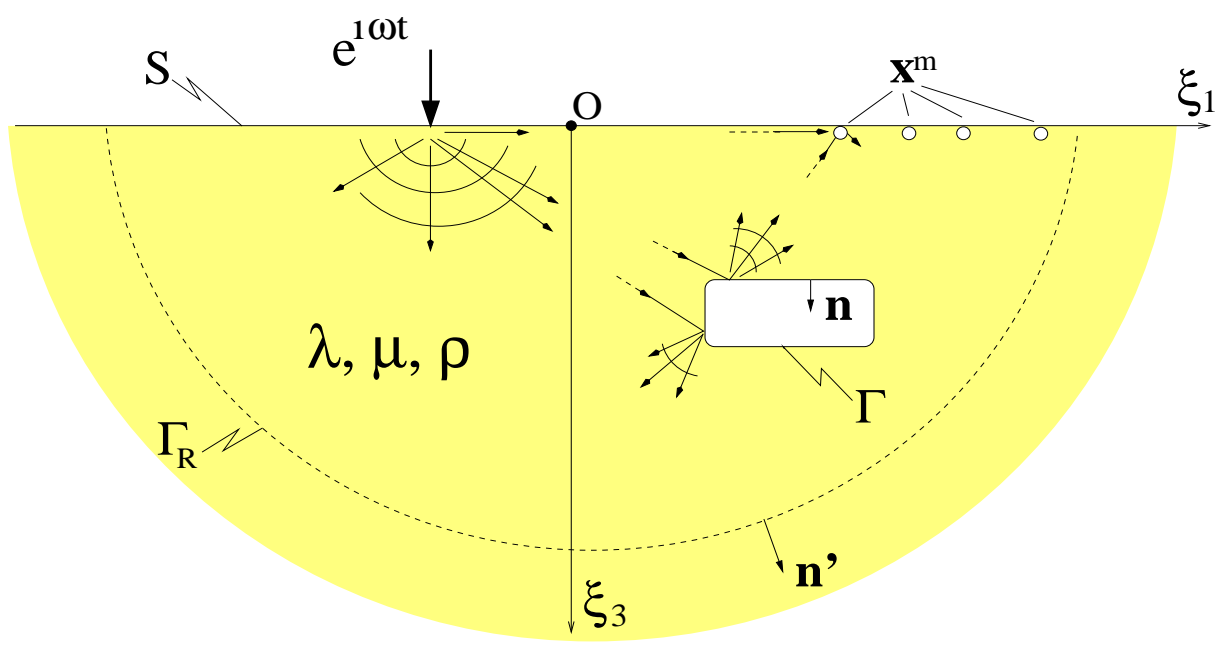

Figure 1: Illumination of an underground cavity by elastic waves

In (1) and (2), $\boldsymbol{f}$ is the time-harmonic body force distribution representing the seismic source; $\boldsymbol{t}$ stands for the surface traction, and $\mathbf{C}$ denotes the isotropic elasticity tensor, i.e.

$$
\mathbf{C}=\lambda \mathbf{I}_{2} \otimes \mathbf{I}_{2}+2 \mu \mathbf{I}_{4}
$$

where $\mathbf{I}_{n}$ is the $n$th order identity tensor.

\subsection{Inverse problem}

With reference to the testing configuration outlined in Fig. 1, the inverse problem of cavity identification can be set forth as a task of resolving the cavity shape and location by interpreting the observed response of the excavated half-space $\Omega^{-}$due to prescribed (i.e. known) seismic excitation.

For this class of remote sensing problems, the inverse solution can be formulated by seeking the minimizer $\Gamma$ of the cost function

$$
\mathcal{J}(\Gamma ; \boldsymbol{f})=J(\boldsymbol{u} ; \boldsymbol{f})+\Psi(\Gamma)
$$

where $J$ represents the misfit between experimental observations $\left(\boldsymbol{u}^{o b s}\right)$ and theoretical, i.e. forward predictions $(\boldsymbol{u})$ for an assumed void geometry $\Gamma$, and $\Psi$ is a non-negative set function used to include an a priori information on the shape and location of the cavity. Upon introducing $\Omega_{o b s} \subset \Omega^{-}$as a

finite control volume enclosing the entirety of measurement stations, $J(\boldsymbol{u} ; \boldsymbol{f})$ can be written in general terms as

$$
J(\boldsymbol{u} ; \boldsymbol{f})=\int_{\Omega_{o b s}} \varphi\left(\boldsymbol{u}(\boldsymbol{\xi}, \omega)-\boldsymbol{u}^{o b s}(\boldsymbol{\xi}, \omega)\right) d \Omega_{\xi}
$$


where $\varphi$ is a weighted measure of distance between $\boldsymbol{u}$ and $\boldsymbol{u}^{o b s}$.

In this investigation, (5) is specialized to the least-squares format with a discrete set of $M$ observation points by taking

$$
\begin{aligned}
\varphi\left(\boldsymbol{u}-\boldsymbol{u}^{o b s}\right) & =\frac{1}{2}\left(\overline{\boldsymbol{u}-\boldsymbol{u}^{o b s}}\right) \cdot \boldsymbol{W} \cdot\left(\boldsymbol{u}-\boldsymbol{u}^{o b s}\right), \\
\boldsymbol{W}=\boldsymbol{W}(\boldsymbol{\xi}) & =\sum_{m=1}^{M} W_{i j}^{m} \delta\left(\boldsymbol{\xi}-\boldsymbol{x}^{m}\right) \boldsymbol{e}_{i} \otimes \boldsymbol{e}_{j}, \quad i, j=1,2,3
\end{aligned}
$$

where $\delta$ stands for the three-dimensional Dirac delta function; $\boldsymbol{e}_{j}$ is the unit vector in the $x_{j}$-direction; over-bar symbol denotes the complex conjugation, and $W_{i j}^{m}$ are suitable constants chosen so that they form a Hermitian and positive definite matrix for $m=1,2, \ldots, M$. In (6) and thereafter, the Einstein summation convention is assumed over the spatial coordinate indices. For consistency of the ensuing formulation, $W_{i j}^{m}$ are assumed to have a physical dimension of force per length.

Assuming that the prior information about the cavity under consideration can be synthesized in terms of a closed surface $\Gamma^{p}$ bounding a fixed finite region $\Omega_{\mathrm{C}}^{p} \subset \Omega$, the penalty function $\Psi$ in (4) can be compactly formulated in terms of the Hausdorff distance $H$ (Edgar, 1990) between sets $\Gamma$ and $\Gamma^{p}$

$$
\Psi(\Gamma)=\vartheta H^{2}\left(\Gamma, \Gamma^{p}\right), \quad H\left(\Gamma, \Gamma^{p}\right)=\max \left\{h\left(\Gamma, \Gamma^{p}\right), h\left(\Gamma^{p}, \Gamma\right)\right\}
$$

where $\vartheta$ is a scalar weighting parameter reflecting the quality of prior knowledge, and

$$
h\left(\Gamma, \Gamma^{p}\right)=\max _{\boldsymbol{\xi} \in \Gamma} \min _{\boldsymbol{\zeta} \in \Gamma^{p}}\{(\boldsymbol{\xi}-\boldsymbol{\zeta}) \cdot(\boldsymbol{\xi}-\boldsymbol{\zeta})\}^{1 / 2} .
$$

In (8), function $h\left(\Gamma, \Gamma^{p}\right)$ is called the directed Hausdorff distance from $\Gamma$ to $\Gamma^{p}$; it locates the point $\boldsymbol{\xi} \in \Gamma$ that is farthest from its nearest neighbor $\boldsymbol{\zeta}$ in $\Gamma^{p}$, and measures $\|\boldsymbol{\xi}-\boldsymbol{\zeta}\|$ for these two points. In situations where the prior information on the cavity geometry (e.g. size, depth) is associated with varying degrees of confidence, however, a more refined measure of the misfit between $\Gamma$ and $\Gamma^{p}$ may be required. To address the problem, it is useful to invoke the Lebesgue measure in $\mathcal{R}^{3}$ (i.e. $\int d \Omega_{\xi}$ ) together with the collection of additive set functions $\int \xi_{i} d \Omega_{\xi}$ and $\int \xi_{i} \xi_{j} d \Omega_{\xi}(i, j=1,2,3)$. With such quantities, a resolute alternative to (7) can be introduced via the quadratic form

$$
\Psi(\Gamma)=\frac{1}{2}\left(\boldsymbol{q}-\boldsymbol{q}^{p}\right) \cdot \boldsymbol{G} \cdot\left(\boldsymbol{q}-\boldsymbol{q}^{p}\right)
$$

where $\boldsymbol{G}$ is a symmetric positive definite matrix of weighting coefficients, $\boldsymbol{q}=\boldsymbol{q}(\Gamma), \boldsymbol{q}^{p}=\boldsymbol{q}\left(\Gamma^{p}\right)$, and 


$$
\boldsymbol{q}(\Gamma)=\left(q_{0}, q_{1}, \cdots, q_{9}\right), \quad q_{s}(\Gamma)= \begin{cases}\int_{\Omega_{c}} d \Omega_{\xi}, & s=0 \\ \frac{1}{q_{0}} \int_{\Omega_{c}} \xi_{s} d \Omega_{\xi}, & s=1,2,3 \\ \int_{\Omega_{c}} \sum_{\substack{j=1 \\ j \neq s-3}}^{3}\left(\xi_{j}-q_{j}\right)^{2} d \Omega_{\xi}, & s=4,5,6 \\ \int_{\Omega_{c}} \prod_{\substack{j=1 \\ j \neq s-6}}^{3}\left(\xi_{j}-q_{j}\right) d \Omega_{\xi}, & s=7,8,9\end{cases}
$$

synthesizes the information about the volume $(s=0)$, centroid $(1 \leq s \leq 3)$, and inertia tensor $(4 \leq s \leq 9)$ of the cavity. For the ensuing developments, the entries of $\boldsymbol{q}$ can be further reduced to surface integrals

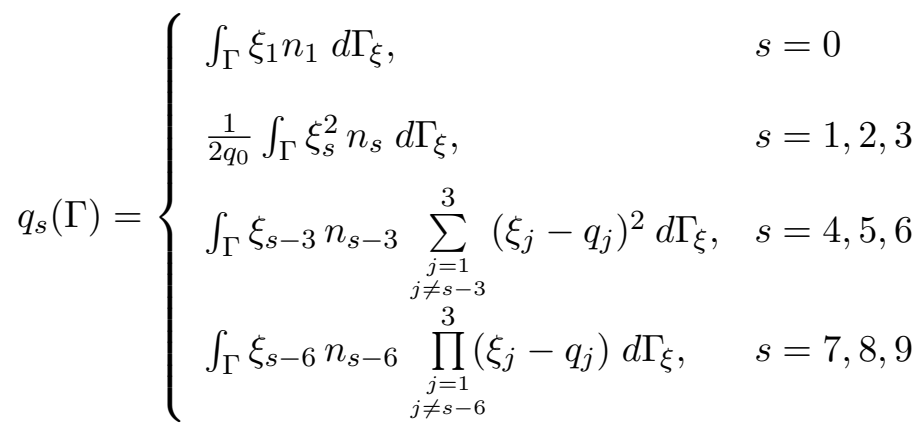

by virtue of the divergence theorem.

In view of the significant computational effort required to evaluate $\boldsymbol{u}$ for elastodynamic problems,

the cost function (4) can be minimized most effectively within the framework of gradient-based descent techniques such as the quasi-Newton or conjugate gradient methods (e.g. Luenberger, 1973).

In the sequel, a systematic derivation of the necessary sensitivities of $\mathcal{J}$ within the framework of boundary integral equation techniques will be described in detail.

\section{Boundary integral formulation for the primary field}

For the computational treatment of (1) and (2), the total displacement field $\boldsymbol{u}$ can be conveniently decomposed as

$$
\boldsymbol{u}=\boldsymbol{u}^{\mathrm{F}}+\boldsymbol{u}^{\mathrm{S}}, \quad \boldsymbol{\xi} \in \Omega^{-},
$$

where $\boldsymbol{u}^{\mathrm{S}}$ denotes the scattered field $\left(\boldsymbol{u}^{\mathrm{S}}=\mathbf{0}\right.$ in the absence of a cavity), and $\boldsymbol{u}^{\mathrm{F}}$ is the free field defined as the response of a cavity-free half-space $\Omega$ due to given body force distribution $f$. By virtue of (1), (2) and (12), it can be shown that the scattered field itself represents an elastodynamic state, i.e. that $\left[\boldsymbol{u}^{\mathrm{S}}, \boldsymbol{t}^{\mathrm{S}}\right]$ satisfies the field equations 


$$
\begin{gathered}
\nabla \cdot \boldsymbol{\sigma}^{\mathrm{S}}=-\rho \omega^{2} \boldsymbol{u}^{\mathrm{S}} \\
\boldsymbol{\sigma}^{\mathrm{S}}=\mathbf{C}: \boldsymbol{\varepsilon}^{\mathrm{S}}, \quad \boldsymbol{\xi} \in \Omega^{-}, \\
\boldsymbol{\varepsilon}^{\mathrm{S}}=\frac{1}{2}\left(\nabla \boldsymbol{u}^{\mathrm{S}}+\nabla^{T} \boldsymbol{u}^{\mathrm{S}}\right),
\end{gathered}
$$

and boundary conditions

$$
\begin{array}{ll}
\boldsymbol{t}^{\mathrm{S}}=-\boldsymbol{t}^{\mathrm{F}}, & \boldsymbol{\xi} \in \Gamma, \\
\boldsymbol{t}^{\mathrm{S}}=\mathbf{0}, & \boldsymbol{\xi} \in S,
\end{array}
$$

with the free field $\left[\boldsymbol{u}^{\mathrm{F}}, \boldsymbol{t}^{\mathrm{F}}\right]$ assumed to be known beforehand.

To obtain an integral representation of $\boldsymbol{u}$ in terms of the boundary data, it is useful to introduce the fundamental solution $[\hat{\boldsymbol{u}}, \hat{\boldsymbol{t}}]$ for a semi-infinite solid $\Omega$, where $\hat{u}_{i}^{k}(\boldsymbol{\xi}, \boldsymbol{x}, \omega)$ and $\hat{t}_{i}^{k}(\boldsymbol{\xi}, \boldsymbol{x}, \omega ; \boldsymbol{n})$ are the respective $i$ th components of the displacement and traction vectors at $\boldsymbol{\xi} \in \Omega$ due to a unit time-harmonic point force acting at $\boldsymbol{x} \in \Omega$ in the $k$ th direction (see also Appendix A). For the ensuing treatment, these Green's functions can be decomposed into a singular part $\left[[\hat{\boldsymbol{u}}]_{1},[\hat{\boldsymbol{t}}]_{1}\right]$ and a residual, i.e. regular component $\left[[\hat{\boldsymbol{u}}]_{2},[\hat{\boldsymbol{t}}]_{2}\right]$ via

$$
\begin{aligned}
& \hat{u}_{i}^{k}(\boldsymbol{\xi}, \boldsymbol{x}, \omega)=\left[\hat{u}_{i}^{k}(\boldsymbol{\xi}, \boldsymbol{x}, \omega)\right]_{1}+\left[\hat{u}_{i}^{k}(\boldsymbol{\xi}, \boldsymbol{x}, \omega)\right]_{2} \equiv \hat{u}_{i}^{k}(\boldsymbol{\xi}, \boldsymbol{x}, 0)+\left[\hat{u}_{i}^{k}(\boldsymbol{\xi}, \boldsymbol{x}, \omega)\right]_{2}, \\
& \hat{t}_{i}^{k}(\boldsymbol{\xi}, \boldsymbol{x}, \omega ; \boldsymbol{n})=\left[\hat{t}_{i}^{k}(\boldsymbol{\xi}, \boldsymbol{x}, \omega ; \boldsymbol{n})\right]_{1}+\left[\hat{t}_{i}^{k}(\boldsymbol{\xi}, \boldsymbol{x}, \omega ; \boldsymbol{n})\right]_{2} \equiv \hat{t}_{i}^{k}(\boldsymbol{\xi}, \boldsymbol{x}, 0 ; \boldsymbol{n})+\left[\hat{t}_{i}^{k}(\boldsymbol{\xi}, \boldsymbol{x}, \omega ; \boldsymbol{n})\right]_{2},
\end{aligned}
$$

where $\hat{\boldsymbol{u}}(\boldsymbol{\xi}, \boldsymbol{x}, 0)$ and $\hat{\boldsymbol{t}}(\boldsymbol{\xi}, \boldsymbol{x}, 0 ; \boldsymbol{n})$ represent the static point-load solution for a void-free elastic half-space $\Omega$ (Guzina and Pak, 2001).

With the foregoing definitions, it can be shown (see Pak and Guzina, 1999) that the scattering problem (1) and (2) can be reformulated in terms of the regularized (i.e. Cauchy principal value-free) boundary integral equation

$$
\begin{aligned}
& \int_{\Gamma}\left\{u_{i}(\boldsymbol{y}, \omega)-u_{i}(\boldsymbol{\xi}, \omega)\right\}\left[\hat{t}_{i}^{k}(\boldsymbol{\xi}, \boldsymbol{y}, \omega ; \boldsymbol{n})\right]_{1} d \Gamma_{\xi} \\
&-\int_{\Gamma} u_{i}(\boldsymbol{\xi}, \omega)\left[\hat{t}_{i}^{k}(\boldsymbol{\xi}, \boldsymbol{y}, \omega ; \boldsymbol{n})\right]_{2} d \Gamma_{\xi}+u_{k}^{\mathrm{F}}(\boldsymbol{y}, \omega)=u_{k}(\boldsymbol{y}, \omega), \quad \boldsymbol{y} \in \Gamma,
\end{aligned}
$$

with the effects of seismic excitation, $\boldsymbol{f}$, synthesized via the free-field term

$$
u_{k}^{\mathrm{F}}(\boldsymbol{y}, \omega)=\int_{\Omega^{-}} f_{i}(\boldsymbol{\xi}) \hat{u}_{i}^{k}(\boldsymbol{\xi}, \boldsymbol{x}, \omega) d \Gamma_{\xi},
$$

given in terms of the half-space fundamental solution. It is important to observe that (16) rests on the a priori assumption that the scattered field satisfies the generalized radiation condition

$$
\lim _{R \rightarrow \infty} \int_{\Gamma_{R}}\left\{u_{i}^{\mathrm{S}}(\boldsymbol{\xi}, \omega) \hat{t}_{i}^{k}\left(\boldsymbol{\xi}, \boldsymbol{x}, \omega ; \boldsymbol{n}^{\prime}\right)-t_{i}^{\mathrm{S}}\left(\boldsymbol{\xi}, \omega ; \boldsymbol{n}^{\prime}\right) \hat{u}_{i}^{k}(\boldsymbol{\xi}, \boldsymbol{x}, \omega)\right\} d \Gamma_{\xi}=0, \quad \boldsymbol{x} \in \Omega_{R}^{-}
$$


where $\boldsymbol{n}^{\prime}$ is the unit normal to $\Gamma_{R}$ directed towards the exterior of $\Omega_{R}^{-}$as indicated in Fig. 1 . Although the far-field requirements of type (18) are implicit to most boundary integral analyses of elastodynamic problems involving semi-infinite domains, they have so far eluded an in-depth scrutiny. For a rigorous pursuit of the forward scattering problem and the associated imaging task, an explicit proof of (18) is the focus of the following section.

\subsection{Generalized radiation condition}

In dealing with the radiation condition for unbounded elastic media, a useful point of departure is the Graffi's reciprocal theorem in elastodynamics (Wheeler and Sternberg, 1968). With reference to an arbitrary finite domain $D$ bounded by surface $\partial D$ with outward normal $\boldsymbol{n}^{\prime}$, Graffi's theorem can be formally stated as

$\int_{\partial D}\left\{u_{i}(\boldsymbol{\xi}, \omega) \tilde{t}_{i}\left(\boldsymbol{\xi}, \omega ; \boldsymbol{n}^{\prime}\right)-t_{i}\left(\boldsymbol{\xi}, \omega ; \boldsymbol{n}^{\prime}\right) \tilde{u}_{i}(\boldsymbol{\xi}, \omega)\right\} d S=\int_{D}\left\{\tilde{f}_{i}(\boldsymbol{\xi}, \omega) u_{i}(\boldsymbol{\xi}, \omega)-\tilde{u}_{i}(\boldsymbol{\xi}, \omega) f_{i}(\boldsymbol{\xi}, \omega)\right\} d V$,

where $[\boldsymbol{u}, \boldsymbol{t}]$ and $[\tilde{\boldsymbol{u}}, \tilde{\boldsymbol{t}}]$ are two arbitrary elastodynamic states on $D$ associated with time-harmonic body force fields $\boldsymbol{f}$ and $\tilde{\boldsymbol{f}}$, respectively. Upon specifying $D=\Omega_{R}, \boldsymbol{f}=\delta(\boldsymbol{\xi}-\boldsymbol{x}) \boldsymbol{e}_{j}$, and $\tilde{\boldsymbol{f}}=\delta(\boldsymbol{\xi}-\boldsymbol{y}) \boldsymbol{e}_{k}$ where $\boldsymbol{x}, \boldsymbol{y} \in \Omega_{R}$ and $\boldsymbol{e}_{j}$ is the unit vector in the $x_{j}$-direction, (19) can be reduced to

$$
\begin{aligned}
& \int_{\Gamma_{R}}\left\{\hat{u}_{i}^{j}(\boldsymbol{\xi}, \boldsymbol{x}, \omega) \hat{t}_{i}^{k}\left(\boldsymbol{\xi}, \boldsymbol{y}, \omega ; \boldsymbol{n}^{\prime}\right)-\hat{t}_{i}^{j}\left(\boldsymbol{\xi}, \boldsymbol{x}, \omega ; \boldsymbol{n}^{\prime}\right) \hat{u}_{i}^{k}(\boldsymbol{\xi}, \boldsymbol{y}, \omega)\right\} d \Gamma_{\xi}= \\
& \hat{u}_{k}^{j}(\boldsymbol{y}, \boldsymbol{x}, \omega)-\hat{u}_{j}^{k}(\boldsymbol{x}, \boldsymbol{y}, \omega), \quad \boldsymbol{x}, \boldsymbol{y} \in \Omega_{R}, \quad j, k \in\{1,2,3\}
\end{aligned}
$$

in terms of the fundamental solution $[\hat{\boldsymbol{u}}, \hat{\boldsymbol{t}}]$ for a void-free elastic half-space $\Omega$. Owing to the intrinsic symmetry of the displacement Green's function: $\hat{u}_{k}^{j}(\boldsymbol{y}, \boldsymbol{x}, \omega)=\hat{u}_{j}^{k}(\boldsymbol{x}, \boldsymbol{y}, \omega)$ as shown in Appendix A, the right-hand side of (20) vanishes so that

$\mathcal{Z}^{j k}(\boldsymbol{x}, \boldsymbol{y} ; R) \equiv \int_{\Gamma_{R}}\left\{\hat{u}_{i}^{j}(\boldsymbol{\xi}, \boldsymbol{x}, \omega) \hat{t}_{i}^{k}\left(\boldsymbol{\xi}, \boldsymbol{y}, \omega ; \boldsymbol{n}^{\prime}\right)-\hat{t}_{i}^{j}(\boldsymbol{\xi}, \boldsymbol{x}, \omega) \hat{u}_{i}^{k}\left(\boldsymbol{\xi}, \boldsymbol{y}, \omega ; \boldsymbol{n}^{\prime}\right)\right\} d \Gamma_{\xi}=0, \quad \boldsymbol{x}, \boldsymbol{y} \in \Omega_{R}$,

for any pair $\boldsymbol{x}, \boldsymbol{y} \in \Omega$ and $R$ sufficiently large so that both $\boldsymbol{x}$ and $\boldsymbol{y}$ are contained within $\Omega_{R}$. It is important to observe that taking the limit of $\mathcal{Z}^{j k}(\boldsymbol{x}, \boldsymbol{y} ; R)$ as $R \rightarrow \infty$ constitutes an explicit proof that the fundamental solution itself satisfies the generalized radiation condition.

To demonstrate the validity of (18) on the basis of (21), it is instructive to represent the scattered field $\boldsymbol{u}^{\mathrm{S}}$ induced by the Neumann boundary conditions (14a) via a single-layer potential (e.g.

Kupradze, 1965)

$$
u_{i}^{\mathrm{S}}(\boldsymbol{x}, \omega)=\int_{\Gamma} g_{j}(\boldsymbol{\zeta}, \omega) \hat{u}_{j}^{i}(\boldsymbol{\zeta}, \boldsymbol{x}, \omega) d \Gamma_{\zeta}, \quad \boldsymbol{x} \in \Omega^{-},
$$

where $\boldsymbol{g}$ denotes an appropriate surface density over $\Gamma$. On employing (22) and interchanging the order of integration, one finds that 


$$
\begin{aligned}
\int_{\Gamma_{R}}\left\{u_{i}^{\mathrm{S}}(\boldsymbol{\xi}, \omega) \hat{t}_{i}^{k}\left(\boldsymbol{\xi}, \boldsymbol{x}, \omega ; \boldsymbol{n}^{\prime}\right)-t_{i}^{\mathrm{S}}\left(\boldsymbol{\xi}, \omega ; \boldsymbol{n}^{\prime}, \Gamma\right) \hat{u}_{i}^{k}(\boldsymbol{\xi}, \boldsymbol{x}, \omega)\right\} d \Gamma_{\xi} & = \\
\int_{\Gamma} g_{j}(\boldsymbol{\zeta}, \omega) \mathcal{Z}^{j k}(\boldsymbol{\zeta}, \boldsymbol{x} ; R) \mathrm{d} \Gamma_{\zeta} & =0, \quad \boldsymbol{x} \in \Omega_{R}^{-},
\end{aligned}
$$

with the latter integral vanishing by virtue of (21) and the postulate that $R$ is sufficiently large so that $\Omega_{R}$ contains the cavity, i.e. that

$$
\left.\begin{array}{c}
\boldsymbol{x} \in \Omega_{R}^{-} \\
\boldsymbol{\zeta} \in \Gamma
\end{array}\right\} \quad \Longrightarrow \quad \boldsymbol{x}, \boldsymbol{\zeta} \in \Omega_{R} .
$$

Upon taking the limit of (23) as $R \rightarrow \infty$, the proof of (18) immediately follows.

In a similar fashion, it can be shown that any elastodynamic field $[\tilde{\boldsymbol{u}}, \tilde{\boldsymbol{t}}]$ in $\Omega^{-}$which admits the integral representation

$$
\tilde{u}_{i}(\boldsymbol{x}, \omega)=\int_{\Gamma} \tilde{g}_{j}(\boldsymbol{\zeta}, \omega) \hat{u}_{j}^{i}(\boldsymbol{\zeta}, \boldsymbol{x}, \omega) d \Gamma_{\zeta}+\int_{\Omega_{b}} \tilde{f}_{j}(\boldsymbol{\zeta}, \omega) \hat{u}_{j}^{i}(\boldsymbol{\zeta}, \boldsymbol{x}, \omega) d \Omega_{\zeta}, \quad \boldsymbol{x} \in \Omega^{-}
$$

in terms of a single-layer potential with density $\tilde{\boldsymbol{g}}$ and a volumetric potential with density $\tilde{\boldsymbol{f}}$ also satisfies the generalized radiation condition, provided that the body force distribution $\tilde{f}$ is confined to a finite region $\Omega_{b} \subset \Omega^{-}$. Finally, it should be noted that the following identity

$$
\lim _{R \rightarrow \infty} \int_{\Gamma_{R}}\left\{t_{i}\left(\boldsymbol{\xi}, \omega ; \boldsymbol{n}^{\prime}\right) \tilde{u}_{i}(\boldsymbol{\xi}, \omega)-u_{i}(\boldsymbol{\xi}, \omega) \tilde{t}_{i}\left(\boldsymbol{\xi}, \omega ; \boldsymbol{n}^{\prime}\right)\right\} d \Gamma_{\xi}=0, \quad \boldsymbol{x} \in \Omega_{R}^{-}
$$

is valid for any two elastodynamic states $[\boldsymbol{u}, \boldsymbol{t}]$ and $[\tilde{\boldsymbol{u}}, \tilde{\boldsymbol{t}}]$ which independently satisfy the generalized radiation condition (18).

\section{Differentiation with respect to shape perturbations}

To investigate the effect of cavity perturbations on the cost function $\mathcal{J}$, the shape $\Omega^{-}$is assumed to depend on a pseudo-time parameter $\tau$ through an Eulerian-type continuum kinematics description. The reference, i.e. unperturbed configuration $\Omega^{-}$is conventionally associated with $\tau=0$, so that the featured domain evolution can be stated as

$$
\boldsymbol{\xi} \in \Omega^{-} \Longrightarrow \boldsymbol{\xi}^{\tau}=\boldsymbol{\Phi}(\boldsymbol{\xi}, \tau) \in \Omega^{-}(\tau), \quad \tau \geq 0, \quad \boldsymbol{\Phi}(\boldsymbol{\xi}, 0)=\boldsymbol{\xi},
$$

where $\boldsymbol{\xi}^{\tau}$ describes the "current" place of the material element $d \mathcal{M}$ that occupied position $\boldsymbol{\xi}$ in the reference configuration. It should be noted that the choice of the geometric transformation $\mathbf{\Phi}$ (with a strictly positive Jacobian) for a specific problem is non-unique, i.e. that a given domain evolution considered as a whole admits infinitely many different representations of type (27). In the ensuing exposition, all pseudo-time derivatives $d(\cdot) / d \tau$ will be implicitly taken at $\tau=0$, i.e. the first-order effect of infinitesimal perturbations of $\Omega^{-} \equiv \Omega^{-}(0)$ will be considered. 


\subsection{Scalar and vector fields}

Differentiation of field variables and integrals with respect to domain perturbation is a well-documented subject, see, e.g. Petryk and Mróz (1986) and Sokolowski and Zolesio (1992). In what follows, several basic concepts and results which are relevant to the study are summarized. To this end, it is instructive to introduce the initial transformation velocity

$$
\boldsymbol{\theta}(\boldsymbol{\xi})=\left.\frac{\partial \boldsymbol{\Phi}}{\partial \tau}\right|_{\tau=0}
$$

Considering the inherently Eulerian description $f(\boldsymbol{\xi}, \tau)$ of a field quantity $f$ in a geometrical transformation, it is natural to define $d f / d \tau$ as its material (i.e. substantial) derivative at $\tau=0$ so

that

$$
\begin{aligned}
\frac{d f}{d \tau} \equiv \stackrel{\star}{f} & =\lim _{\tau \rightarrow 0} \frac{1}{\tau}\left\{f\left(\boldsymbol{\xi}^{\tau}, \tau\right)-f(\boldsymbol{\xi}, 0)\right\} \\
& =f^{\prime}+\nabla f \cdot \boldsymbol{\theta}
\end{aligned}
$$

where $f^{\prime}=\partial f / \partial \tau$ stands for the local rate of change, i.e. partial "time" derivative with $\boldsymbol{\xi}$ kept fixed, and $\nabla$ implies differentiation with respect to Eulerian coordinates $\boldsymbol{\xi}$. Similarly, the material derivative of the gradient of $f$ is given by

$$
(\nabla f)^{\star}=\nabla \stackrel{\star}{f}-\nabla f \cdot \nabla \boldsymbol{\theta}
$$

\subsection{Volume and surface integrals}

With reference to the continuity equation (e.g. Malvern, 1969)

$$
\stackrel{\star}{\mathrm{d}} V=(\nabla \cdot \boldsymbol{\theta}) \mathrm{d} V
$$

describing the volume evolution of a given material element $d \mathcal{M}$ under geometric transformation (27), the material derivative of a generic volume integral

$$
I_{V}(f, D ; \tau)=\int_{D(\tau)} f(\boldsymbol{\xi}, \tau) \mathrm{d} V
$$

can be expressed via either of the following two statements

$$
\begin{aligned}
\frac{d I_{V}}{d \tau} \equiv \stackrel{\star}{I}_{V} & =\int_{D}\{\stackrel{\star}{f}+f \nabla \cdot \boldsymbol{\theta}\} \mathrm{d} V \\
& =\int_{D} f^{\prime} \mathrm{d} V+\int_{\partial D} f \theta_{n} \mathrm{~d} S
\end{aligned}
$$

of the classical Reynolds formula where $\theta_{n}=\boldsymbol{\theta} \cdot \boldsymbol{n}$. 
To formulate the counterpart of (32) for surface integrals, one must allow for kernels which may be undefined outside of a given material surface $S(\tau)$. To this end, it is useful to introduce the concepts of surface gradient $\left(\nabla_{S}\right)$ and surface divergence $\left(\nabla_{S} \cdot\right)$ via

$$
\begin{aligned}
\nabla_{S} f & =\nabla f-(\nabla f \cdot \boldsymbol{n}) \boldsymbol{n}=\left(f_{, i}-n_{i} f_{, n}\right) \boldsymbol{e}_{i} \equiv\left(D_{i} f\right) \boldsymbol{e}_{i}, \\
\nabla_{S} \cdot \boldsymbol{u} & =\nabla \cdot \boldsymbol{u}-(\nabla \boldsymbol{u} \cdot \boldsymbol{n}) \cdot \boldsymbol{n}=D_{i} u_{i},
\end{aligned}
$$

which characterize the tangential variation of the respective fields along $S$. With such definitions, material derivatives of the unit normal $\boldsymbol{n}$ and the differential element $\mathrm{d} S$ on a moving surface $S(\tau)$ can be written as (e.g. Petryk and Mróz, 1986)

$$
\stackrel{\star}{\mathrm{d}} S=\left(\nabla_{S} \cdot \boldsymbol{\theta}\right) \mathrm{d} S=D_{i} \theta_{i} \mathrm{~d} S, \quad \stackrel{\star}{\boldsymbol{n}}=-\boldsymbol{n} \cdot \nabla_{S} \boldsymbol{\theta}=-n_{j} D_{i} \theta_{j} \boldsymbol{e}_{i}
$$

By virtue of (34a), the material derivative of a generic surface integral

$$
I_{S}(f, S ; \tau)=\int_{S(\tau)} f(\boldsymbol{\xi}, \tau) \mathrm{d} S
$$

can be shown to permit the representation

$$
\frac{d I_{S}}{d \tau} \equiv \stackrel{\star}{I}_{S}=\int_{S}\left\{\stackrel{\star}{f}+f \nabla_{S} \cdot \boldsymbol{\theta}\right\} \mathrm{d} S
$$

in terms of the initial transformation velocity. Upon combining eqs. (34), one may also find that

$$
\left(n_{i} \mathrm{~d} S\right)^{\star}=\left(n_{i} D_{j}-n_{j} D_{i}\right) \theta_{j} \mathrm{~d} S \equiv D_{i j} \theta_{j} \mathrm{~d} S
$$

which results in the following two variants of (35) involving products of $\boldsymbol{n}$ and scalar or vector fields:

$$
\begin{aligned}
\frac{d}{d \tau} \int_{S} f n_{i} \mathrm{~d} S & =\int_{S}\left\{\stackrel{\star}{f} n_{i}+f D_{i j} \theta_{j}\right\} \mathrm{d} S \\
\frac{d}{d \tau} \int_{S} u_{i} n_{i} \mathrm{~d} S & =\int_{S}\left\{u_{i}^{\prime} n_{i}+D_{i j}\left(u_{i} \theta_{j}\right)+\theta_{n} u_{i, i}\right\} \mathrm{d} S .
\end{aligned}
$$

As shown in Bonnet (1995a), the operator $D_{i j}$ in (36) and (37) is a tangential differential operator which, by virtue of the Stokes' theorem, satisfies the identity

$$
\int_{S} D_{i j} f \mathrm{~d} S=-\int_{\partial S} e_{i j k}\left(e_{l m k} f\right)_{, m} n_{l} \mathrm{~d} S=0
$$

for any closed surface $S$ where $e_{i j k}$ denotes the permutation symbol. On the basis of (37b) and (38),

the equality

$$
\frac{d}{d \tau} \int_{S} \boldsymbol{u} \cdot \boldsymbol{n} \mathrm{d} S=\int_{S}\left(\boldsymbol{u}^{\prime} \cdot \boldsymbol{n}+\theta_{n} \nabla \cdot \boldsymbol{u}\right) \mathrm{d} S
$$


immediately follows. As a consequence, upon setting $\boldsymbol{u}=\boldsymbol{w} \cdot \boldsymbol{\sigma}$ where $\boldsymbol{w}$ and $\boldsymbol{\sigma}$ denote arbitrary elastodynamic displacement and stress fields, respectively, one has

$$
\begin{aligned}
\frac{d}{d \tau} \int_{S} \boldsymbol{w} \cdot \boldsymbol{t} \mathrm{d} S & =\int_{S}\left\{\boldsymbol{w}^{\prime} \cdot \boldsymbol{t}+\boldsymbol{w} \cdot\left(\boldsymbol{\sigma}^{\prime} \cdot \boldsymbol{n}\right)+\theta_{n} \nabla \cdot(\boldsymbol{w} \cdot \boldsymbol{\sigma})\right\} \mathrm{d} S \\
& =\int_{S}\left\{\boldsymbol{w}^{\prime} \cdot \boldsymbol{t}+\boldsymbol{w} \cdot\left(\boldsymbol{\sigma}^{\prime} \cdot \boldsymbol{n}\right)+\theta_{n}(\boldsymbol{\sigma}: \nabla \boldsymbol{w}+(\nabla \cdot \boldsymbol{\sigma}) \cdot \boldsymbol{w})\right\} \mathrm{d} S
\end{aligned}
$$

where $\boldsymbol{t}=\boldsymbol{\sigma} \cdot \boldsymbol{n}$ stands for the surface traction.

\section{Shape differentiation using an adjoint solution}

As examined earlier, a convenient approach to the nonlinear minimization of (4) with respect to $\Gamma$ involves gradient search (e.g. modified Newton) methods which inherently require repeated

evaluation of $\stackrel{\star}{\mathcal{J}}$ due to cavity shape perturbations. In view of the well-known computational drawbacks of the finite-difference estimators, a common practice in the sensitivity analysis of functionals such as $\mathcal{J}$ is to employ an adjoint problem approach (e.g. Choi and Kwak, 1988; Bonnet, 1995a) which, besides the matter of elegance, combines the computational accuracy and efficiency unmatched by numerical differentiation techniques. In the present context involving unbounded media, however, a direct application of this method may lead to ambiguities associated with the conditions at infinity. To resolve the puzzle, a rigorous treatment of the adjoint problem-based sensitivity formula for unbounded elastic solids with an emphasis on the generalized radiation condition (18) is the focus of this section.

\subsection{Augmented functional}

For an effective minimization of $\mathcal{J}(\Gamma ; \boldsymbol{f})$, it is useful to employ the method of Lagrange multipliers and treat the field equations (1) and boundary conditions (2) satisfied by the primary field $\boldsymbol{u}$ as constraints. Accordingly, the constraints on $\boldsymbol{u}$ are introduced in the form of a weak statement over the domain $\Omega_{R}^{-}$:

$$
\mathcal{A}_{R}(\Gamma, \boldsymbol{u}, \tilde{\boldsymbol{u}} ; \boldsymbol{f})=\int_{\Omega_{R}^{-}}\left(\nabla \cdot \boldsymbol{\sigma}+\boldsymbol{f}+\rho \omega^{2} \boldsymbol{u}\right) \cdot \tilde{\boldsymbol{u}} \mathrm{d} \Omega-\int_{\Gamma+S_{R}} \boldsymbol{t} \cdot \tilde{\boldsymbol{u}} \mathrm{d} \Gamma=0
$$

where $\Omega_{R}^{-}$is centered at the origin and the Lagrange multiplier $\tilde{\boldsymbol{u}}$ belongs to a space of test functions $\mathcal{V}=\left\{\tilde{\boldsymbol{u}} \in\left\{H_{l o c}^{1}\left(\Omega^{-}\right)\right\}^{3}\right\}$. On integrating (41) by parts, it can be shown that

$$
\begin{gathered}
\mathcal{A}_{R}(\Gamma, \boldsymbol{u}, \tilde{\boldsymbol{u}} ; \boldsymbol{f})=\int_{\Omega_{R}^{-}}\left(\nabla \cdot(\mathbf{C}: \nabla \tilde{\boldsymbol{u}})+\rho \omega^{2} \tilde{\boldsymbol{u}}\right) \cdot \boldsymbol{u} \mathrm{d} \Omega+\int_{\Omega_{R}^{-}} \boldsymbol{f} \cdot \tilde{\boldsymbol{u}} \mathrm{d} \Omega \\
+\int_{\Gamma_{R}}(\tilde{\boldsymbol{u}} \cdot \boldsymbol{t}-\boldsymbol{u} \cdot \tilde{\boldsymbol{t}}) \mathrm{d} \Gamma-\int_{\Gamma+S_{R}} \tilde{\boldsymbol{t}} \cdot \boldsymbol{u} \mathrm{d} \Gamma
\end{gathered}
$$


where $\Gamma_{R}$ and $S_{R}$ are the surfaces bounding $\Omega_{R}^{-}$as elucidated before, and $\tilde{\boldsymbol{t}}=(\mathbf{C}: \nabla \tilde{\boldsymbol{u}}) \cdot \boldsymbol{n}$. It is important to observe that the limit of (42) as $R$ tends to infinity represents a weak formulation of (1) and (2) with $\tilde{\boldsymbol{u}}$ used as a weighting field. As a result, under the assumption that

$$
\lim _{R \rightarrow \infty} \int_{\Gamma_{R}}(\tilde{\boldsymbol{u}} \cdot \boldsymbol{t}-\boldsymbol{u} \cdot \tilde{\boldsymbol{t}}) \mathrm{d} \Gamma=0
$$

which will be demonstrated later, one finds that

$$
\begin{aligned}
\mathcal{A}(\Gamma, \boldsymbol{u}, \tilde{\boldsymbol{u}} ; \boldsymbol{f}) & \equiv \lim _{R \rightarrow \infty} \mathcal{A}_{R}(\Gamma, \boldsymbol{u}, \tilde{\boldsymbol{u}} ; \boldsymbol{f}) \\
& =\int_{\Omega^{-}}\left(\nabla \cdot(\mathbf{C}: \nabla \tilde{\boldsymbol{u}})+\rho \omega^{2} \tilde{\boldsymbol{u}}\right) \cdot \boldsymbol{u} \mathrm{d} \Omega+\int_{\Omega^{-}} \boldsymbol{f} \cdot \tilde{\boldsymbol{u}} \mathrm{d} \Omega-\int_{\Gamma+S} \tilde{\boldsymbol{t}} \cdot \boldsymbol{u} \mathrm{d} \Gamma=0 .
\end{aligned}
$$

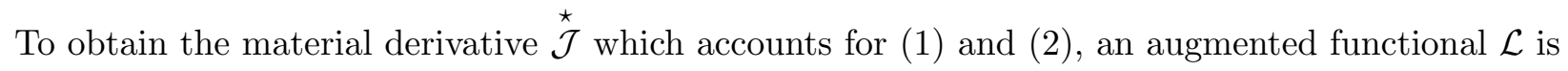
introduced on the basis of (44) where

$$
\mathcal{L}(\Gamma, \boldsymbol{u}, \tilde{\boldsymbol{u}} ; \boldsymbol{f})=J(\boldsymbol{u} ; \boldsymbol{f})+\Psi(\Gamma)+\operatorname{Re}[\mathcal{A}(\Gamma, \boldsymbol{u}, \tilde{\boldsymbol{u}} ; \boldsymbol{f})]
$$

In what follows, it will be assumed without loss of generality that (i) the transformation velocity (28) vanishes near the observation points $\boldsymbol{x}^{m}(m=1,2, \ldots M)$ which are by definition away from the cavity; (ii) the free surface of the half-space remains flat in a geometric transformation so that

$$
\begin{aligned}
\boldsymbol{\theta}(\boldsymbol{\xi}) & =\mathbf{0}, & & \boldsymbol{\xi} \in \Omega_{o b s}, \\
\theta_{n}(\boldsymbol{\xi}) & \equiv \boldsymbol{\theta}(\boldsymbol{\xi}) \cdot \boldsymbol{n}=0, & & \boldsymbol{\xi} \in S,
\end{aligned}
$$

where $\boldsymbol{n}$ is the outward normal to $\Omega^{-}$, and (iii) the prior information $\Psi$ is specified via (9). By virtue of (4), (6), (10), (32) and the foregoing assumptions, the material derivative of $\mathcal{L}$ can be expressed as

$$
\begin{aligned}
& \stackrel{\star}{\mathcal{L}}=\stackrel{\star}{J}+\stackrel{\star}{\Psi}+\operatorname{Re}[\stackrel{\star}{\mathcal{A}}]=\operatorname{Re}\left[\int_{\Omega_{o b s}}\left(\overline{\boldsymbol{u}-\boldsymbol{u}^{o b s}}\right) \cdot \boldsymbol{W} \cdot \boldsymbol{u}^{\prime} \mathrm{d} \Omega+\left(\boldsymbol{q}-\boldsymbol{q}^{p}\right) \cdot \boldsymbol{G} \cdot \stackrel{\star}{\boldsymbol{q}}^{*} \stackrel{\star}{\mathcal{A}}\right] \\
& \text { where } \boldsymbol{u}^{\prime}=\partial \boldsymbol{u} / \partial \tau, \text { and } \\
& \stackrel{\star}{\boldsymbol{q}}(\Gamma)=\left(\stackrel{\star}{q}_{0}, \stackrel{\star}{q}_{1}, \cdots, \stackrel{\star}{q}\right), \quad \stackrel{\star}{q}_{s}(\Gamma)= \begin{cases}\int_{\Gamma} \theta_{n} d \Gamma_{\xi}, & s=0 \\
\frac{1}{q_{0}} \int_{\Gamma}\left(\xi_{s}-q_{s}\right) \theta_{n} d \Gamma_{\xi}, & s=1,2,3 \\
\int_{\Gamma} \sum_{\substack{j=1 \\
j \neq s-3}}^{3}\left(\xi_{j}-q_{j}\right)^{2} \theta_{n} d \Gamma_{\xi}, & s=4,5,6 \\
\int_{\Gamma} \prod_{\substack{j=1 \\
j \neq s-6}}^{3}\left(\xi_{j}-q_{j}\right) \theta_{n} d \Gamma_{\xi}, & s=7,8,9\end{cases}
\end{aligned}
$$

with $q_{s}(s=0,1, \cdots, 9)$ given by (11) in terms of surface integrals over $\Gamma$. 
For the remote sensing problem of interest, it is natural to postulate that the support of $\boldsymbol{f}$ which is used to "illuminate" the cavity lies entirely outside of $\Omega_{\mathrm{C}}$, i.e. that $\boldsymbol{f}(\boldsymbol{\xi}) \equiv \mathbf{0}$ for $\boldsymbol{\xi} \in \Gamma$. With such

hypothesis and the fundamental property of the test function space

$$
\begin{gathered}
\mathcal{A}(\Gamma, \boldsymbol{u}, \tilde{\boldsymbol{u}} ; \boldsymbol{f})=0 \Longrightarrow \mathcal{A}\left(\Gamma, \boldsymbol{u}, \tilde{\boldsymbol{u}}^{\prime} ; \boldsymbol{f}\right)=0, \quad \forall \tilde{\boldsymbol{u}} \in \mathcal{V}, \\
\qquad \begin{array}{c}
\stackrel{\star}{\mathcal{L}}(\Gamma, \boldsymbol{u}, \tilde{\boldsymbol{u}} ; \boldsymbol{f})=\operatorname{Re}\left[\int_{\Omega^{-}}\left(\nabla \cdot \tilde{\boldsymbol{\sigma}}+\left(\overline{\boldsymbol{u}-\boldsymbol{u}^{o b s}}\right) \cdot \boldsymbol{W}+\rho \omega^{2} \tilde{\boldsymbol{u}}\right) \cdot \boldsymbol{u}^{\prime} \mathrm{d} \Omega-\int_{\Gamma+S} \tilde{\boldsymbol{t}} \cdot \boldsymbol{u}^{\prime} \mathrm{d} \Gamma\right] \\
+\operatorname{Re}\left[\int_{\Gamma}\left(\rho \omega^{2} \tilde{\boldsymbol{u}} \cdot \boldsymbol{u}-\tilde{\boldsymbol{\sigma}}: \nabla \boldsymbol{u}\right) \theta_{n} \mathrm{~d} \Gamma\right]+\left(\boldsymbol{q}-\boldsymbol{q}^{p}\right) \cdot \boldsymbol{G} \cdot \stackrel{\star}{\boldsymbol{q}}
\end{array}
\end{gathered}
$$

where $\tilde{\boldsymbol{\sigma}}=\mathbf{C}: \nabla \tilde{\boldsymbol{u}}$, and $\stackrel{\star}{\boldsymbol{q}}$ is homogeneous in $\theta_{n}$ as specified by (48).

\section{$5.2 \quad$ Adjoint state}

Following the conventional approach in the theory of optimization, the Lagrange multiplier $\tilde{\boldsymbol{u}}$ is chosen so that $\stackrel{\star}{\mathcal{L}}$ vanishes when the normal transformation velocity $\theta_{n}=0$, i.e. that the fist term in (50) equals zero. Such a requirement directly defines the (elastodynamic) adjoint state $\tilde{\boldsymbol{u}}$ as a solution to the variational problem

$$
\int_{\Omega^{-}}\left(\nabla \cdot \tilde{\boldsymbol{\sigma}}+\left(\overline{\boldsymbol{u}-\boldsymbol{u}^{o b s}}\right) \cdot \boldsymbol{W}+\rho \omega^{2} \tilde{\boldsymbol{u}}\right) \cdot \boldsymbol{u}^{\prime} \mathrm{d} \Omega-\int_{\Gamma+S} \tilde{\boldsymbol{t}} \cdot \boldsymbol{u}^{\prime} \mathrm{d} \Gamma=0
$$

whose strong statement can be written explicitly in terms of the field equations

$$
\begin{gathered}
\nabla \cdot \tilde{\boldsymbol{\sigma}}+\boldsymbol{W} \cdot\left(\overline{\boldsymbol{u}-\boldsymbol{u}^{o b s}}\right)=-\rho \omega^{2} \tilde{\boldsymbol{u}}, \\
\tilde{\boldsymbol{\sigma}}=\mathbf{C}: \tilde{\boldsymbol{\varepsilon}}, \quad \boldsymbol{\xi} \in \mathbf{\Omega}^{-}, \\
\tilde{\boldsymbol{\varepsilon}}=\frac{1}{2}\left(\nabla \tilde{\boldsymbol{u}}+\nabla^{T} \tilde{\boldsymbol{u}}\right), \\
\tilde{\boldsymbol{t}}=\mathbf{0}, \quad \boldsymbol{\xi} \in \Gamma \cup S .
\end{gathered}
$$

and boundary conditions

Given the primary field $\boldsymbol{u}$ for an assumed cavity geometry, the solution to (52) and (53) can be effectively evaluated in terms of the regularized boundary integral equation (16) with the free field $u_{k}^{\mathrm{F}}$ taken as the response of a cavity-free half-space due to internal sources $\tilde{\boldsymbol{f}}=\boldsymbol{W} \cdot\left(\overline{\boldsymbol{u}-\boldsymbol{u}^{\text {obs }}}\right)$ which are proportional to the misfit between experimental observations and forward predictions at measurement locations.

On the basis of (1), (25) and (52), it can further be shown that both the primary field and the adjoint state satisfy the generalized radiation condition owing to the localized (i.e. finite) support of the respective body force distributions, $\boldsymbol{f}$ and $\tilde{\boldsymbol{f}}$. With such observation and lemma (26), the proof of the relationship (43) (whose validity was assumed earlier) immediately follows. 


\subsection{Gradient formula}

In view of $(50),(51)$ and the identity $\stackrel{\star}{\mathcal{A}}=0$, the material (i.e. shape) derivative of the cost function (4) reduces to

$$
\stackrel{\star}{\mathcal{J}}(\Gamma ; \boldsymbol{f})=\stackrel{\star}{\mathcal{L}}(\Gamma, \boldsymbol{u}, \tilde{\boldsymbol{u}} ; \boldsymbol{f})=\operatorname{Re}\left[\int_{\Gamma}\left(\rho \omega^{2} \tilde{\boldsymbol{u}} \cdot \boldsymbol{u}-\tilde{\boldsymbol{\sigma}}: \nabla \boldsymbol{u}\right) \theta_{n} \mathrm{~d} \Gamma\right]+\left(\boldsymbol{q}-\boldsymbol{q}^{p}\right) \cdot \boldsymbol{G} \cdot \stackrel{\star}{\boldsymbol{q}}
$$

where $\boldsymbol{u}$ and $\tilde{\boldsymbol{u}}$ are the forward and adjoint solutions, respectively. This formula is similar to that obtained by Bonnet (1995a) for a homogeneous full-space problem without an account for the radiation condition.

Despite its elegance, however, expression (54) may not be tractable within the framework of boundary integral techniques owing to the difficulties associated with the evaluation of total displacement gradients at the boundary (e.g. Sladek and Sladek, 1986). To circumvent such impediment, it is instructive to invoke the concept of surface gradients (33) and observe that

$$
\begin{aligned}
\tilde{\boldsymbol{\sigma}}: \nabla \boldsymbol{u} & =\tilde{\boldsymbol{\sigma}}:\left(\nabla_{S} \boldsymbol{u}+(\nabla \boldsymbol{u} \cdot \boldsymbol{n}) \otimes \boldsymbol{n}\right) \\
& =\tilde{\boldsymbol{\sigma}}: \nabla_{S} \boldsymbol{u}+\tilde{\boldsymbol{t}} \cdot \boldsymbol{u}_{, n}
\end{aligned}
$$

where $\boldsymbol{u}_{, n}=\nabla \boldsymbol{u} \cdot \boldsymbol{n}$. By means of (55) and taking advantage of the Neumann boundary condition (53), material derivative (54) can be rewritten as

$$
\stackrel{\star}{\mathcal{J}}(\Gamma ; \boldsymbol{f})=\operatorname{Re}\left[\int_{\Gamma}\left(\rho \omega^{2} \tilde{\boldsymbol{u}} \cdot \boldsymbol{u}-\tilde{\boldsymbol{\sigma}}: \nabla_{S} \boldsymbol{u}\right) \theta_{n} d \Gamma\right]+\left(\boldsymbol{q}-\boldsymbol{q}^{p}\right) \cdot \boldsymbol{G} \cdot \stackrel{\star}{\boldsymbol{q}}
$$

Finally, on expressing $\tilde{\boldsymbol{\sigma}}$ in terms of $\tilde{\boldsymbol{t}}$ and $\tilde{\boldsymbol{u}}_{, n}$ for an isotropic elastic solid (see Bonnet, 1995b), (56) can be shown to permit the representation

$$
\begin{aligned}
& \stackrel{\star}{\mathcal{J}}(\Gamma ; \boldsymbol{f})=\operatorname{Re}\left[\int _ { \Gamma } \left\{\rho \omega^{2} \tilde{\boldsymbol{u}} \cdot \boldsymbol{u}-\frac{2 \lambda \mu}{\lambda+2 \mu}\left(\nabla_{S} \cdot \tilde{\boldsymbol{u}}\right)\left(\nabla_{S} \cdot \boldsymbol{u}\right)-\mu\left(\nabla_{S} \tilde{\boldsymbol{u}}+\nabla_{S}^{T} \tilde{\boldsymbol{u}}\right): \nabla_{S} \boldsymbol{u}\right.\right. \\
& \left.\left.+\mu\left(\boldsymbol{n} \cdot \nabla_{S} \tilde{\boldsymbol{u}}\right) \cdot\left(\boldsymbol{n} \cdot \nabla_{S} \boldsymbol{u}\right)\right\} \theta_{n} d \Gamma\right]+\left(\boldsymbol{q}-\boldsymbol{q}^{p}\right) \cdot \boldsymbol{G} \cdot \stackrel{\star}{\boldsymbol{q}},
\end{aligned}
$$

which involves strictly the tangential derivatives that are readily computable from the nodal values of $\boldsymbol{u}$ and $\tilde{\boldsymbol{u}}$ on $\Gamma$.

\subsection{Additional considerations}

To demonstrate the generality of (45) in view of the fact that the imaginary part of $\mathcal{A}$ is neglected in constructing the augmented functional, it is important to observe that taking $\mathcal{L}$ in the alternative form

$$
\mathcal{L}(\Gamma, \boldsymbol{u}, \tilde{\boldsymbol{u}} ; \boldsymbol{f})=J(\boldsymbol{u} ; \boldsymbol{f})+\Psi(\Gamma)+\operatorname{Im}[\mathcal{A}(\Gamma, \boldsymbol{u}, \tilde{\boldsymbol{u}} ; \boldsymbol{f})]
$$


yields the expression for $\stackrel{\star}{\mathcal{J}}$ which is identical to the formula derived earlier on the basis of (45). As a result the shape derivative (57), with the featured adjoint state given by (52) and (53), intrinsically enforces both real and imaginary components of the weak statement (44) as required by the inverse solution.

In situations when a set of $K$ sequential seismic fields, generated by the respective time-harmonic body force distributions $\boldsymbol{f}^{k}(k=1,2, \cdots, K)$ is used to illuminate the cavity, the foregoing developments can be generalized by writing

$\mathcal{J}\left(\Gamma ; \boldsymbol{f}^{1}, \cdots, \boldsymbol{f}^{K}\right)=\frac{1}{2} \sum_{k=1}^{K} \int_{\Omega_{o b s}}\left(\overline{\boldsymbol{u}^{k}-\boldsymbol{u}^{k, o b s}}\right) \cdot \boldsymbol{W}^{k} \cdot\left(\boldsymbol{u}^{k}-\boldsymbol{u}^{k, o b s}\right) d \Omega_{\xi}+\frac{1}{2}\left(\boldsymbol{q}-\boldsymbol{q}^{p}\right) \cdot \boldsymbol{G} \cdot\left(\boldsymbol{q}-\boldsymbol{q}^{p}\right)$,

and

$$
\begin{array}{r}
\stackrel{\star}{\mathcal{J}}\left(\Gamma ; \boldsymbol{f}^{1}, \cdots, \boldsymbol{f}^{K}\right)=\sum_{k=1}^{K} \operatorname{Re}\left[\int _ { \Gamma } \left\{\rho \omega_{k}^{2} \tilde{\boldsymbol{u}}^{k} \cdot \boldsymbol{u}^{k}-\frac{2 \lambda \mu}{\lambda+2 \mu}\left(\nabla_{S} \cdot \tilde{\boldsymbol{u}}^{k}\right)\left(\nabla_{S} \cdot \boldsymbol{u}^{k}\right)-\mu\left(\nabla_{S} \tilde{\boldsymbol{u}}^{k}+\nabla_{S}^{T} \tilde{\boldsymbol{u}}^{k}\right): \nabla_{S} \boldsymbol{u}^{k}\right.\right. \\
\left.\left.+\mu\left(\boldsymbol{n} \cdot \nabla_{S} \tilde{\boldsymbol{u}}^{k}\right) \cdot\left(\boldsymbol{n} \cdot \nabla_{S} \boldsymbol{u}^{k}\right)\right\} \theta_{n} d \Gamma\right]+\left(\boldsymbol{q}-\boldsymbol{q}^{p}\right) \cdot \boldsymbol{G} \cdot \stackrel{\star}{\boldsymbol{q}}, \quad(60)
\end{array}
$$

for the cost function and its material derivative, respectively. In (60), $\left[\tilde{\boldsymbol{u}}^{k}, \tilde{\boldsymbol{t}}^{k} \equiv \tilde{\boldsymbol{\sigma}}^{k} \cdot \boldsymbol{n}\right]$ denotes the adjoint elastodynamic state associated with the $k$ th seismic source where

$$
\begin{gathered}
\nabla \cdot \tilde{\boldsymbol{\sigma}}^{k}+\boldsymbol{W}^{k} \cdot\left(\overline{\boldsymbol{u}^{k}-\boldsymbol{u}^{k, o b s}}\right)=-\rho \omega_{k}^{2} \tilde{\boldsymbol{u}}^{k}, \\
\tilde{\boldsymbol{\sigma}}^{k}=\mathbf{C}: \tilde{\boldsymbol{\varepsilon}}^{k}, \quad \boldsymbol{\xi} \in \Omega^{-}, \\
\tilde{\boldsymbol{\varepsilon}}^{k}=\frac{1}{2}\left(\nabla \tilde{\boldsymbol{u}}^{k}+\nabla^{T} \tilde{\boldsymbol{u}}^{k}\right), \\
\tilde{\boldsymbol{t}}^{k}=\mathbf{0}, \quad \boldsymbol{\xi} \in \Gamma \cup S .
\end{gathered}
$$

\section{Computational treatment and results}

In practice, the location and shape of $\Gamma$ is taken to depend on a finite set of design parameters, $\boldsymbol{p}=\left(p_{1}, p_{2}, \ldots, p_{D}\right)$. With such assumption, the sensitivity formulas $\partial \mathcal{J} / \partial p_{d}$ required for the minimization of $\mathcal{J}$ can be obtained by setting $\tau=p_{d}(d=1,2, \ldots, D)$ in (60). As long as the topological characteristics of $\Gamma$ are independent of $\tau$ (e.g. $\Gamma(\tau)$ remains simply connected), the evolving boundary element mesh representing $\Gamma(\tau)$ can be created by interpolating a suitable set of parameter-dependent nodes $\boldsymbol{x}^{q}(\boldsymbol{p})$ with fixed, i.e. pre-defined mesh connectivity. For a generic point

$\xi \in \Gamma$ and a given $Q$-noded boundary element $E \subset \Gamma$, the foregoing interpolation can be formally

written as

$$
\boldsymbol{\xi}(\boldsymbol{p})=\sum_{q=1}^{Q} N_{q}(\boldsymbol{\eta}) \boldsymbol{x}^{q}(\boldsymbol{p}), \quad \boldsymbol{\xi} \in E, \quad \boldsymbol{\eta} \in E_{0},
$$


where $N_{q}(\boldsymbol{\eta})$ are the shape functions for the $Q$-noded element with parent domain $E_{0}$. With (63), the boundary element solution is implemented on the basis of (16) in a standard fashion. For the gradient-based back-analysis, each partial derivative $\partial \mathcal{J} / \partial p_{d}$ is computed by applying (60) with the transformation velocity $\boldsymbol{\theta}=\boldsymbol{\theta}^{d}$ given by

$$
\boldsymbol{\theta}^{d}=\sum_{q=1}^{Q} N_{q}(\boldsymbol{\eta}) \frac{\partial \boldsymbol{x}^{q}}{\partial p_{d}}(\boldsymbol{p}), \quad \boldsymbol{\xi} \in E, \quad \boldsymbol{\eta} \in E_{0} .
$$

In this investigation, surface of the cavity is discretized via eight-node quadratic boundary elements (see, e.g., Brebbia et al., 1984).

\subsection{Gradient evaluation}

To illustrate the performance of the adjoint problem approach, a numerical experiment was performed with reference to the spherical cavity pictured in Fig. 2 whose design parameters,

$$
\boldsymbol{p}=\left(p_{1}, p_{2}, p_{3}\right) \text {, represent the position of its center. In this case, (64) reduces to }
$$

$$
\boldsymbol{\theta}^{d}=\boldsymbol{e}_{d}, \quad d=1,2,3
$$

The true and trial cavities are centered respectively at $\boldsymbol{p}^{\text {true }}=(0,0,2 a)$ and $\boldsymbol{p}^{0}=(2 a, 3 a, 6 a)$, where $a$ denotes the radius of the sphere. The testing configuration shown in the Figure has nine points; in

succession, each grid node is taken as a location of the vertical point source, with the remaining eight points used as receivers, so that a total of $K \times M \times 3=9 \times 8 \times 3=216$ synthetic observation data are generated for the true cavity. The constitutive parameters of the half-space and the testing frequency are chosen such that

$$
\frac{\lambda}{\mu}=\frac{3}{2}, \quad \bar{\omega} \equiv \frac{\omega a}{\sqrt{\mu / \rho}}=1 .
$$

To expose the performance of the adjoint problem approach, objective function for the trial cavity is computed via (4) to (6) with $W_{i j}^{m}=10^{6} \mu a \delta_{i j}$ and no prior information, i.e. $\Psi=0$.

In Table 1, rows 3 through 6 show a comparison between the adjoint approach and central difference estimates in terms of the sensitivities $\partial \mathcal{J} / \partial p_{d}(d=1,2,3)$ for an assumed location of the trial cavity.

The step size for the finite difference calculation is taken as $\Delta p_{d}=0.002 a$. As can be seen from the Table, there is a reasonable agreement between the two methods. It should be noted, however, that the computational time for the adjoint approach is approximately $1 / 6$ of that for the central difference method in the problem examined. For a general setting involving $D$ design parameters, the foregoing efficiency ratio can be estimated as $1 /(2 D)$ since the central difference method requires $2 D$ computations of the BIE (16), each corresponding to a different (perturbed) configuration of $\Gamma$.

In the foregoing example, the initial transformation velocity is restricted to a constant value, i.e. $\boldsymbol{\theta}=\boldsymbol{e}_{d}(d=1,2,3)$ as driven by the assumed parameterization. To investigate the performance of $(60)$ 


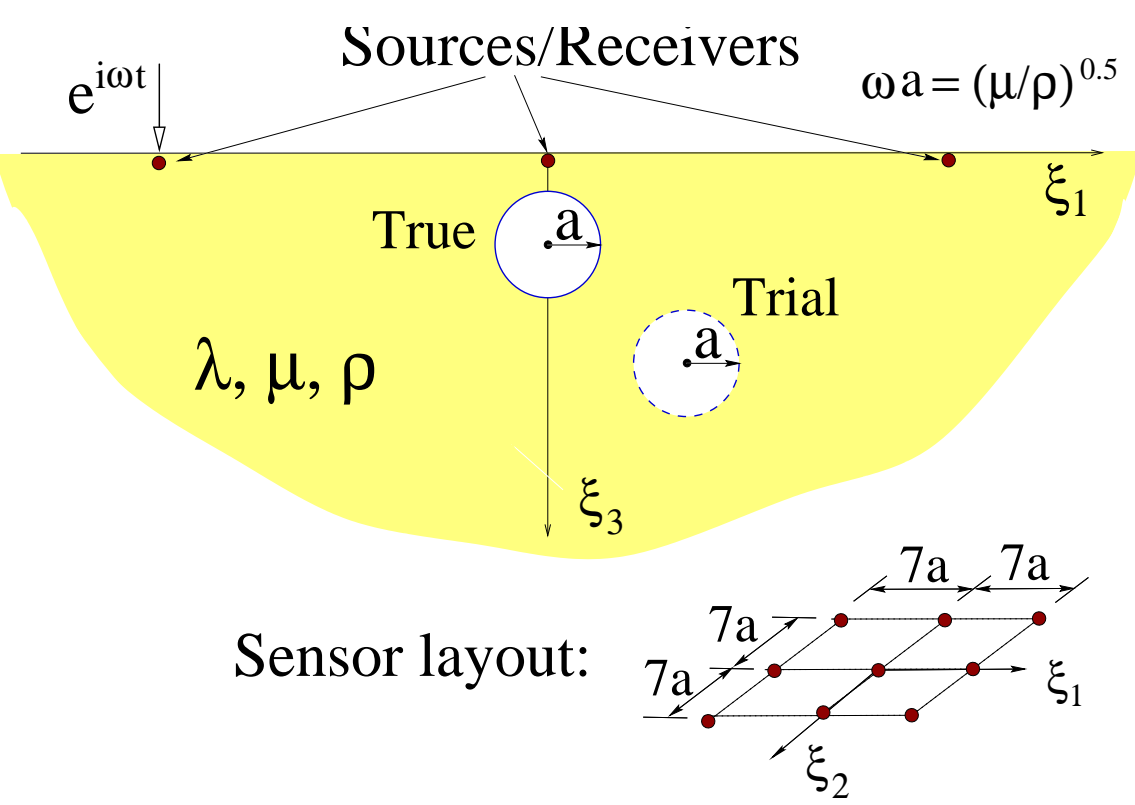

Figure 2: Spherical cavity and testing grid in a half-space with $\lambda / \mu=3 / 2$

Table 1: Gradient comparison: finite difference vs. adjoint approach

\begin{tabular}{|c||c|c||c|c|}
\hline \hline Mesh & \multicolumn{2}{|c||}{96 elements, $\mathcal{J}=61.62 \mu a^{3}$} & \multicolumn{2}{|c|}{294 elements, $\mathcal{J}=61.99 \mu a^{3}$} \\
\hline Method & Central diff. & Adjoint & Central diff. & Adjoint \\
\hline \hline $1 /\left(\mu a^{2}\right) \partial \mathcal{J} / \partial p_{1}$ & 0.8858 & 0.8761 & 0.8764 & 0.8779 \\
\hline $1 /\left(\mu a^{2}\right) \partial \mathcal{J} / \partial p_{2}$ & 1.4184 & 1.4322 & 1.4431 & 1.4350 \\
\hline $1 /\left(\mu a^{2}\right) \partial \mathcal{J} / \partial p_{3}$ & -1.6642 & -1.6549 & -1.6715 & -1.6703 \\
\hline \hline $1 /\left(\mu a^{2}\right) \stackrel{\jmath}{\mathcal{J}}$ & 2.2762 & 2.2363 & 2.2720 & 2.2543 \\
\hline \hline
\end{tabular}

under more general conditions, the finite difference and adjoint approach estimates of $\stackrel{\star}{\mathcal{J}}$ are further compared in the last row of Table 1 for the case

$$
\boldsymbol{\theta}(\boldsymbol{\xi})=\sin \frac{\xi_{1}}{a} \sin \frac{\xi_{2}}{a} \log _{e} \frac{\xi_{3}}{a} \boldsymbol{e}_{1}+\frac{\xi_{1} \xi_{2}}{a^{2}} \sin \frac{\pi \xi_{3}}{a} \boldsymbol{e}_{2}+\frac{\xi_{1} \xi_{2}^{2}}{a^{3}} e^{-\xi_{3} / a} \boldsymbol{e}_{3},
$$

with the finite difference approximation calculated as

$$
\stackrel{\star}{\mathcal{J}} \approx \frac{\mathcal{J}\left(\Gamma+\tau \boldsymbol{\theta} ; \boldsymbol{f}^{1}, \cdots, \boldsymbol{f}^{9}\right)-\mathcal{J}\left(\Gamma-\tau \boldsymbol{\theta} ; \boldsymbol{f}^{1}, \cdots, \boldsymbol{f}^{9}\right)}{2 \tau}, \quad \tau=0.002 a
$$

From the Table, an overall agreement between the two methods should again be apparent. 


\subsection{Cavity imaging}

The next example deals with the inverse scattering problem for an ellipsoidal void illustrated in Fig. 3, whose semi-axes are aligned with the global coordinate system. For imaging purposes, the cavity is parameterized in terms of its centroid coordinates $c_{i}$ and semi-axes lengths $r_{i}(i=1,2,3)$ so

that

$$
\boldsymbol{p}=\left(c_{1}, c_{2}, c_{3}, r_{1}, r_{2}, r_{3}\right)
$$

The true void geometry, with volume $V^{\text {true }}=4.072 a^{3}$, is given by $\boldsymbol{p}^{\text {true }}=(-4 a,-2 a, 4 a, 1.8 a, 0.9 a, 0.6 a)$; its trial counterpart is taken as $\boldsymbol{p}^{0}=(-2 a, 0,5 a, a, a, a)$. The cavity is illuminated in succession via nine point sources according to the testing grid depicted in Fig. 2. For each incident seismic field, Cartesian components of the surface motion are monitored at

64 control points uniformly spaced over the square observation area $(14 a \times 14 a)$ bounded by the source grid. Similar to the previous example, the constitutive parameters and testing frequency are chosen after (66). From the problem configuration, one may observe that the shear wave length of the illuminating seismic field is approximately twice the largest diameter of the true cavity.

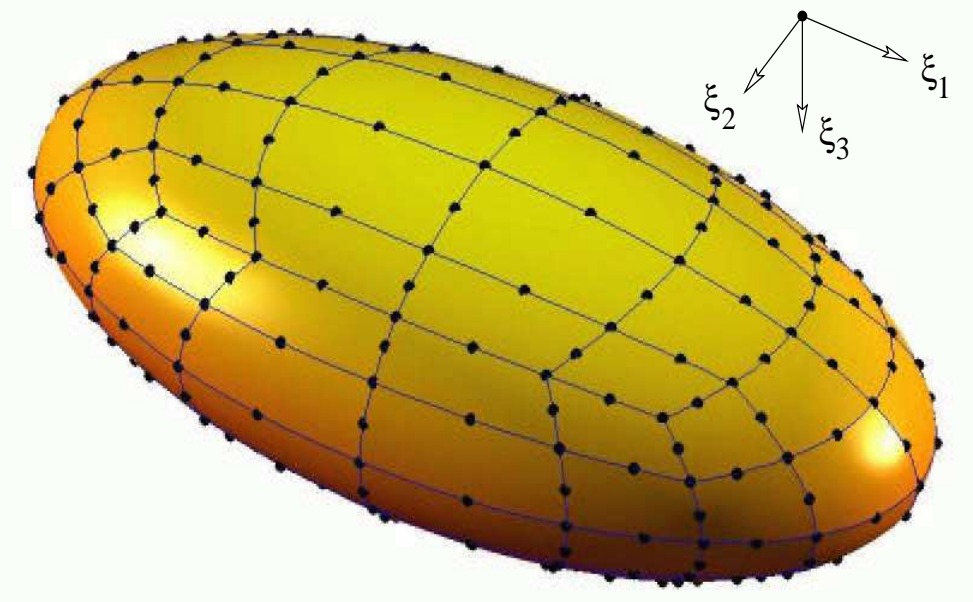

Figure 3: Surface of an ellipsoidal cavity discretized via 96 eight-noded boundary elements

With reference to (4), (5), (6) and (9), the cost function $\mathcal{J}$ is computed with $W_{i j}^{m}=10^{6} \mu a \delta_{i j}$ and the prior information on the cavity geometry given by

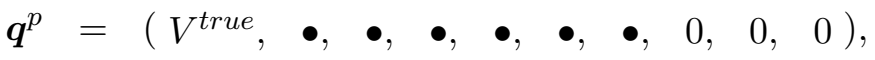

$$
\begin{aligned}
& \boldsymbol{G}=2 \times 10^{-6} \mu \operatorname{diag}\left[a^{-3}, \quad 0, \quad 0, \quad 0, \quad 0, \quad 0, \quad 0, a^{-7}, a^{-7}, a^{-7}\right],
\end{aligned}
$$

which indicate an opening of volume $V=V^{\text {true }}$ whose principal axes of inertia are aligned with the global coordinate system. In (70), indicated by the bullet symbol are the entries of $\boldsymbol{q}$ (with zero 
weighting coefficient) on which no prior information is available, namely the cavity's centroid coordinates and its principal moments of inertia. One may also observe that the last three entries of $\boldsymbol{q}^{p}$, while formally constituting the prior information, have been already assimilated into the problem via parameterization (69). As a result, the only limitation directly enforced through (70) is that on the cavity volume, with the weighting coefficient $\left(2 \times 10^{-6} \mu\right)$ chosen so that the prior knowledge component in (4) is an order of magnitude larger than its misfit counterpart, $J$, at $\boldsymbol{p}=\boldsymbol{p}^{0}$. To simulate the presence of modeling and measurement uncertainties, synthetic observations of the ground motion $\left(\boldsymbol{u}^{o b s}\right)$ are contaminated with the aid of a perturbation factor $(1+\varrho)$ applied to their scattered component, where $\varrho$ is a random variable uniformly distributed over the interval $[-0.01,0.01]$. The Matlab minimization procedure, employed in this study, revolves around an unconstrained quasi-Newton descent method with a quadratic line search algorithm, where the Hessian operator is updated via the BFGS formula (see, e.g. Nocedal and Wright, 1999). With reference to the results obtained in Section 6.1, the computational time is reduced by a factor of 12 by estimating the sensitivity functions $\partial \mathcal{J} / \partial p_{d}(d=1,2, \ldots, 6)$ via an adjoint problem approach.

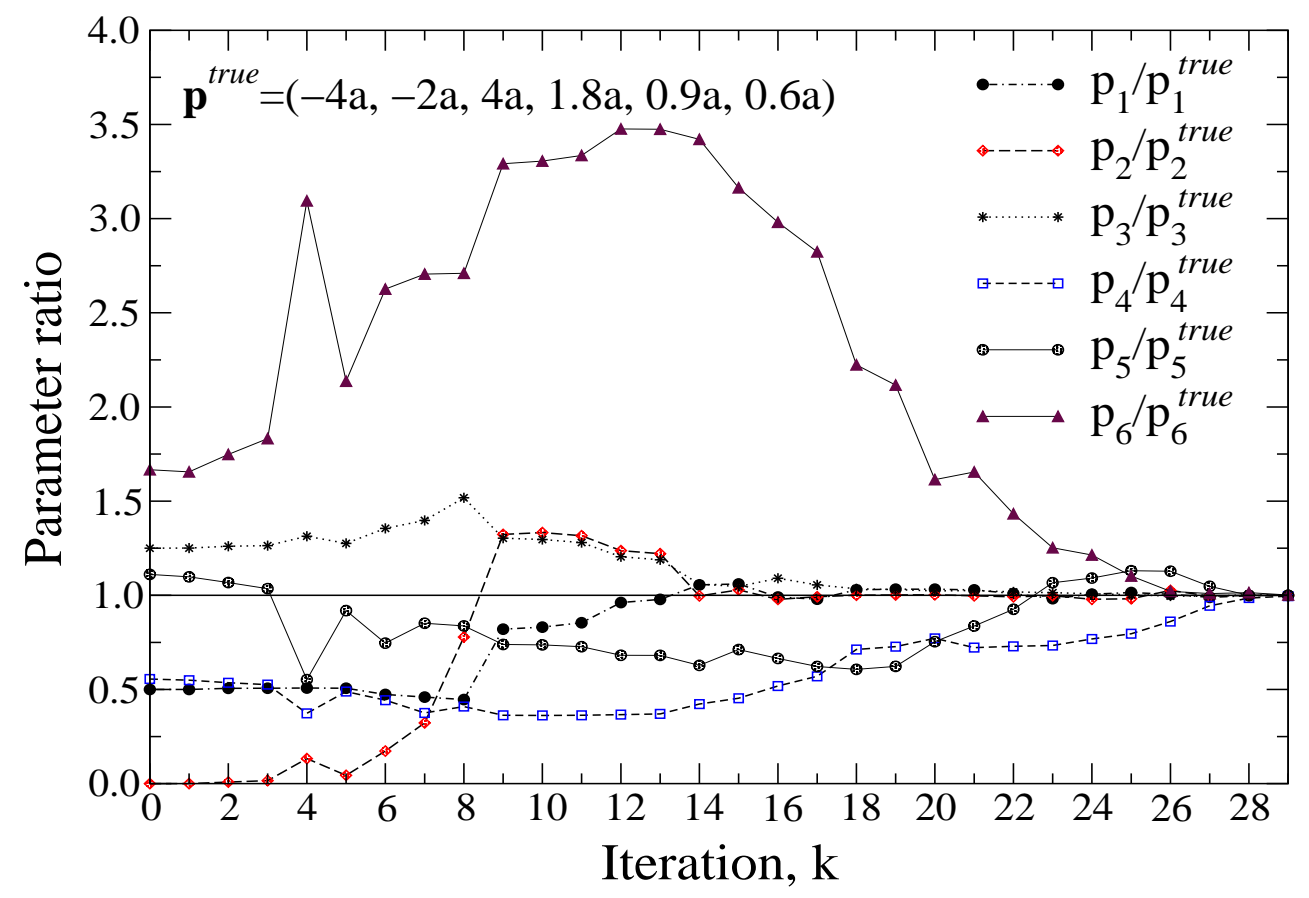

Figure 4: Evolution of design parameters in the minimization process

Fig. 4 illustrates the iterative process of mapping the featured cavity, $\boldsymbol{p}^{\text {true }}$, starting from $\boldsymbol{p}=\boldsymbol{p}^{0}$. As can be seen from the Figure, the optimization procedure converges to the global minimum after approximately thirty major iterations. For clarity, Fig. 5 depicts the imaging procedure geometrically in plane view. It should be noted that the success of the foregoing method is strongly dependent on the choice of a 


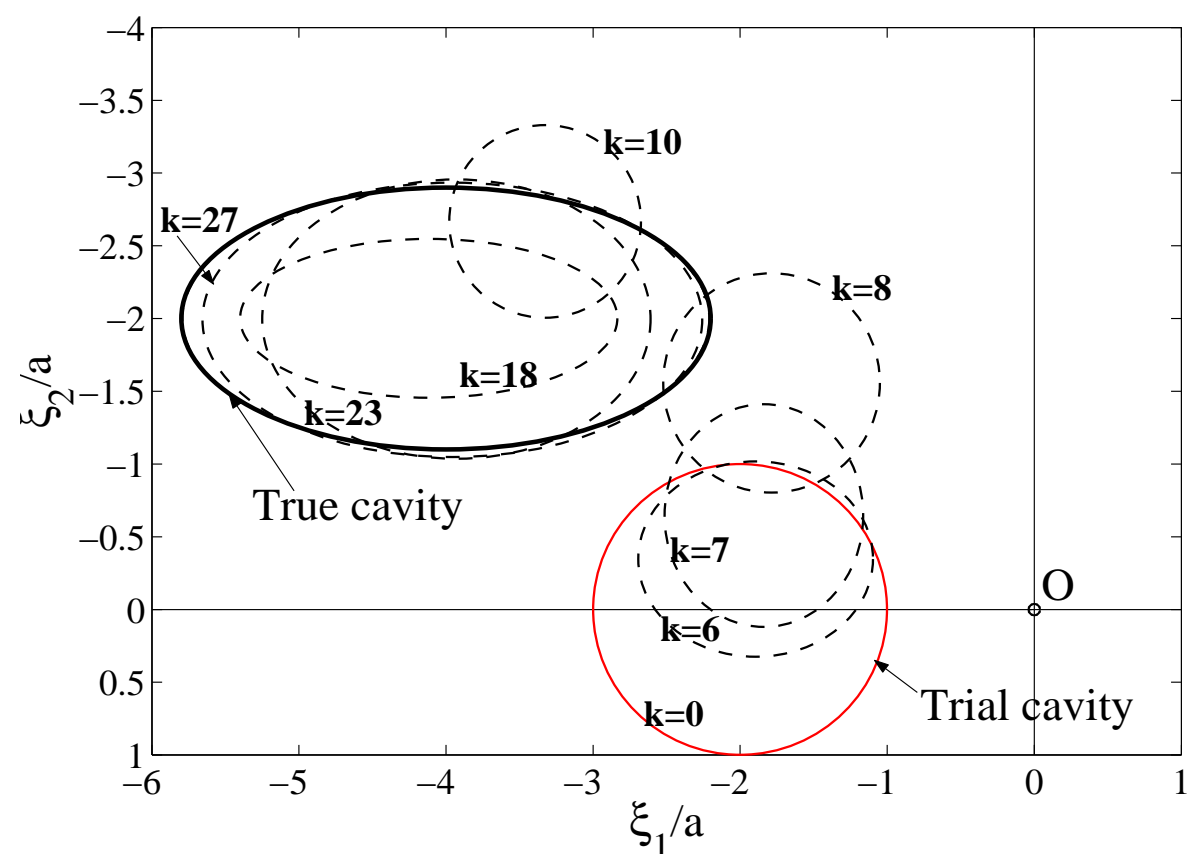

Figure 5: Projection of true and trial cavities on the horizontal plane

starting point, a pitfall that is common to all gradient-based algorithms. Such a deficiency could be alleviated by restarting the search procedure from a variety of initial points or, alternatively, by selecting $\boldsymbol{p}^{0}$ using the concept of topological derivative $\mathcal{T}(\boldsymbol{x})$ (Sokolowski and Zochowski, 1999;

Garreau et al., 2001), which furnishes an information about the variation of the cost function $\mathcal{J}$, when a spherical cavity of infinitesimal radius is introduced at $\boldsymbol{x} \in \Omega$. A more robust, yet reasonably efficient minimization algorithm could be devised by employing a random global search (e.g. a genetic algorithm, Gen and Cheng, 2000), followed by the descent procedure described herein.

\section{Conclusions}

In this communication, the problem of mapping three-dimensional cavities in a semi-infinite solid from surface seismic measurements is investigated via a regularized boundary integral equation

(BIE) method. With the solid modeled as a uniform elastic half-space, the inverse problem is reduced to the gradient-based minimization of a misfit between the observed surface motion and its elastodynamic prediction for an assumed void location. In the formulation, necessary sensitivities of the predictive BIE model are evaluated via an adjoint problem approach and an Eulerian-type continuum kinematics description. A rigorous treatment of the radiation condition for an elastic half-space, which is essential to both forward and inverse scattering problems involving semi-infinite solids, is elucidated. The proposed format of the cost function, which includes weighted measures of

(i) observation-theory misfit and (ii) prior information, further lends itself to stochastic generalizations such as the maximum likelihood inverse theory (Tarantola, 1987). Numerical results 
show that the adjoint problem approach produces sensitivity estimates that are consistent with their finite difference counterparts, while reducing the computational time by a factor of $2 D$, where $D$ is the number of design parameters used to describe the cavity geometry. Beyond serving as an effective tool for the three-dimensional imaging of voids concealed by a uniform semi-infinite solid, the analysis furnishes the basis for extensions of the methodology to problems involving solid inclusions in layered media, with potential use in meso- and micro-scale material characterization, defense applications, and the diagnosis of medical ailments. It is also shown that the elastic waves are capable of resolving inhomogeneities smaller than the predominant wave length of an illuminating wave field, a situation that is considered to be beyond the resolution limits of stress-wave (e.g. ultrasonic) material testing.

\section{Acknowledgment}

The support provided by the National Science Foundation through CAREER Award No. CMS-9875495 to B. Guzina and the University of Minnesota Supercomputing Institute during the course of this investigation is gratefully acknowledged. Special thanks are due to MTS Systems

Corporation for providing the opportunity for M. Bonnet to visit the University of Minnesota through the MTS Visiting Professorship of Geomechanics.

\section{References}

Bonnet, M., 1995a, "BIE and material differentiation applied to the formulation of obstacle inverse problems," Eng. Anal. with Bound. Elem., vol. 15, pp. 121-136.

Bonnet, M., 1995b, "Regularized BIE formulations for first- and second-order shape sensitivity of elastic fields," Comput. ES Struct., vol. 56, pp. 799-811.

Brebbia, C. A., Telles, J. C. F., and Wrobel, L. C., 1984, Boundary element techniques, Springer Verlag.

Bui, H. D., 1994, Inverse problems in the mechanics of materials, CRC Press.

Choi, J. O. and Kwak, B. M., 1988, "Boundary integral equation method for optimization of elastic structures," Int. J. Num. Meth. Eng., vol. 26, pp. 1579-1595.

Colton, D., Coyle, J., and Monk, P., 2000, "Recent developments in inverse acoustic scattering theory." SIAM Review, vol. 42, pp. 369-414.

Colton, D. and Kress, R., 1983, Integral Equation Method in Scattering Theory, Wiley, New York. 
Colton, D. and Kress, R., 1992, Inverse acoustic and electromagnetic scattering theory, Springer Verlag.

Edgar, G. A., 1990, Measure, Topology, and Fractal Geometry, Springer-Verlag, New York.

Garreau, S., Guillaume, P., and Masmoudi, M., 2001, "The topological asymptotic for pde systems: The elasticity case." SIAM J. Control Optim., vol. 39, pp. 1756-1778.

Gen, M. and Cheng, R., 2000, Genetic Algorithms and Engineering Optimization, Wiley-Interscience, New York.

Guzina, B. B. and Pak, R. Y. S., 2001, "On the analysis of wave motions in a multi-layered solid," Quart. J. Mech. Appl. Math., vol. 54, pp. 13-37.

Kress, R., 1996, "Inverse elastic scattering from a crack." Inverse Problems, vol. 12, pp. 667-684.

Kupradze, V. D., 1965, Potential Methods in the Theory of Elasticity, Israel Program for Scientific Translations, Jerusalem.

Luenberger, D. G., 1973, Introduction to Linear and Nonlinear Programming, Addison-Wesley, Reading.

Malvern, L. E., 1969, Introduction to the Mechanics of a Continuous Medium, Prentice-Hall, Englewood Cliffs.

Nishimura, N., 1997, "Crack determination problems," Theoretical and Applied Mechanics, volume 46, eds. G. Yagawa and C. Miki, pp. 39-57.

Nocedal, J. and Wright, S. J., 1999, Numerical Optimization, Springer, New York.

Pak, R. Y. S., 1987, "Asymmetric wave propagation in a half-space by a method of potentials," $J$. Appl. Mech., ASME, vol. 54, pp. 121-126.

Pak, R. Y. S. and Guzina, B. B., 1999, "Seismic soil-tructure interaction analysis by direct boundary element methods," Int. J. Solids Struct., vol. 36, pp. 4743-4766.

Petryk, H. and Mróz, Z., 1986, "Time derivatives of integrals and functionals defined on varying volume and surface domains," Arch. Mech., vol. 38, pp. 694-724.

Pike, R. and Sabatier, P. C., eds., 2002, Scattering and Inverse Scattering in Pure and Applied Science, vol. 1 \& 2, Academic Press, San Diego.

Plessix, R. E., De Roeck, Y. H., and Chavent, G., 1999, "Waveform inversion of reflection seismic data for kinematic parameters by local optimization." SIAM J. Sci. Comput., vol. 20, pp. 1033-1052. 
Sladek, V. and Sladek, J., 1986, "Improved computation of stresses using the boundary element method," Appl. Math. Modelling, vol. 10, pp. 249-255.

Sokolowski, J. and Zochowski, A., 1999, "On the topological derivative in shape optimization." SIAM J. Control Optim., vol. 37, pp. 1251-1272.

Sokolowski, J. and Zolesio, J. P., 1992, Introduction to shape optimization. Shape sensitivity analysis, vol. 16 of Springer series in Computational Mathematics, Springer-Verlag.

Tarantola, A., 1987, Inverse Problem Theory, Elsevier, Amsterdam.

Wheeler, L. T. and Sternberg, E., 1968, "Some theorems in classical elastodynamics," Arch. Rat. Mech. Anal., vol. 31, pp. 51-90. 


\section{Appendix: Symmetry of fundamental solution}

By means of the method of displacement potentials and the Hankel integral transform (see Pak, 1987), it can be shown that the time-harmonic displacement fundamental solution $\hat{u}_{i}^{k}$ for a homogeneous elastic half-space $\Omega$ permits the integral representation

$$
\begin{aligned}
\hat{u}_{1}^{k}(\boldsymbol{x}, \boldsymbol{y}, \omega)= & \frac{1}{4 \pi \mu}\left\{\delta_{k 1} \int_{0}^{\infty}\left(\gamma_{2}+\gamma_{1}\right) \xi J_{0}(r \xi) d \xi+\left(\delta_{k 1} \cos 2 \theta+\delta_{k 2} \sin 2 \theta\right) \times\right. \\
& \left.\left.\int_{0}^{\infty}\left(\gamma_{2}-\gamma_{1}\right) \xi J_{2}(r \xi) d \xi-2 \delta_{k 3} \cos \theta \int_{0}^{\infty} \gamma_{3} \xi J_{1}(r \xi) d \xi\right)\right\}, \\
\hat{u}_{2}^{k}(\boldsymbol{x}, \boldsymbol{y}, \omega)= & \frac{1}{4 \pi \mu}\left\{\delta_{k 2} \int_{0}^{\infty}\left(\gamma_{2}+\gamma_{1}\right) \xi J_{0}(r \xi) d \xi+\left(\delta_{k 1} \sin 2 \theta-\delta_{k 2} \cos 2 \theta\right) \times\right. \\
& \left.\left.\int_{0}^{\infty}\left(\gamma_{2}-\gamma_{1}\right) \xi J_{2}(r \xi) d \xi-2 \delta_{k 3} \sin \theta \int_{0}^{\infty} \gamma_{3} \xi J_{1}(r \xi) d \xi\right)\right\}, \\
\hat{u}_{3}^{k}(\boldsymbol{x}, \boldsymbol{y}, \omega)= & \frac{1}{4 \pi \mu}\left\{\delta_{k 3} \int_{0}^{\infty} \Omega_{2} \xi J_{0}(r \xi) d \xi+\left(\delta_{k 1} \cos \theta+\delta_{k 2} \sin \theta\right) \int_{0}^{\infty} \Omega_{1} \xi J_{1}(r \xi) d \xi\right\},
\end{aligned}
$$

where $\omega$ is the circular frequency of vibration, $k=1,2,3$ denotes the force direction, and $\boldsymbol{x}$ and $\boldsymbol{y}$ are the receiver and source locations, respectively. In (A.1), $\delta$ denotes the Kronecker delta; $J_{n}$ is the

Bessel function of order $n$, and

$$
\begin{aligned}
& r=\sqrt{\left(x_{1}-y_{1}\right)^{2}+\left(x_{2}-y_{2}\right)^{2}}, \quad \cos \theta=\frac{x_{1}-y_{1}}{r}, \\
& \text { with the kernel functions are given by } \\
& \gamma_{1}(\xi ; \boldsymbol{x}, \boldsymbol{y}, \omega)=\frac{\xi^{2}}{2 \alpha k_{s}^{2}} e^{-\alpha\left|d_{1}\right|}-\frac{\beta}{2 k_{s}^{2}} e^{-\beta\left|d_{1}\right|}-\frac{1}{2 k_{s}^{2}} \frac{R^{+}(\xi)}{R^{-}(\xi)}\left(\frac{\xi^{2}}{\alpha} e^{-\alpha d_{2}}+\beta e^{-\beta d_{2}}\right)+ \\
& \frac{2 \xi^{2} \beta\left(\xi^{2}+\beta^{2}\right)}{k_{s}^{2} R^{-}(\xi)}\left(e^{-\left(\beta x_{3}+\alpha y_{3}\right)}+e^{-\left(\beta y_{3}+\alpha x_{3}\right)}\right), \\
& \gamma_{2}(\xi ; \boldsymbol{x}, \boldsymbol{y}, \omega)=\frac{1}{2 \beta}\left(e^{-\beta\left|d_{1}\right|}+e^{-\beta d_{2}}\right), \\
& \gamma_{3}(\xi ; \boldsymbol{x}, \boldsymbol{y}, \omega)=\operatorname{sgn}\left(d_{1}\right) \frac{\xi}{2 k_{s}^{2}}\left(e^{-\alpha\left|d_{1}\right|}-e^{-\beta\left|d_{1}\right|}\right)+\frac{\xi}{2 k_{s}^{2}} \frac{R^{+}(\xi)}{R^{-}(\xi)}\left(e^{-\alpha d_{2}}+e^{-\beta d_{2}}\right)- \\
& \frac{2 \xi\left(\xi^{2}+\beta^{2}\right)}{k_{s}^{2} R^{-}(\xi)}\left(\alpha \beta e^{-\left(\beta x_{3}+\alpha y_{3}\right)}+\xi^{2} e^{-\left(\beta y_{3}+\alpha x_{3}\right)}\right), \\
& \Omega_{1}(\xi ; \boldsymbol{x}, \boldsymbol{y}, \omega)=-\operatorname{sgn}\left(d_{1}\right) \frac{\xi}{2 k_{s}^{2}}\left(e^{-\alpha\left|d_{1}\right|}-e^{-\beta\left|d_{1}\right|}\right)+\frac{\xi}{2 k_{s}^{2}} \frac{R^{+}(\xi)}{R^{-}(\xi)}\left(e^{-\alpha d_{2}}+e^{-\beta d_{2}}\right)- \\
& \frac{2 \xi\left(\xi^{2}+\beta^{2}\right)}{k_{s}^{2} R^{-}(\xi)}\left(\xi^{2} e^{-\left(\beta x_{3}+\alpha y_{3}\right)}+\alpha \beta e^{-\left(\beta y_{3}+\alpha x_{3}\right)}\right), \\
& \Omega_{2}(\xi ; \boldsymbol{x}, \boldsymbol{y}, \omega)=-\frac{\alpha}{2 k_{s}^{2}} e^{-\alpha\left|d_{1}\right|}+\frac{\xi^{2}}{2 \beta k_{s}^{2}} e^{-\beta\left|d_{1}\right|}-\frac{1}{2 k_{s}^{2}} \frac{R^{+}(\xi)}{R^{-}(\xi)}\left(\alpha e^{-\alpha d_{2}}+\frac{\xi^{2}}{\beta} e^{-\beta d_{2}}\right)+ \\
& \frac{2 \xi^{2} \alpha\left(\xi^{2}+\beta^{2}\right)}{k_{s}^{2} R^{-}(\xi)}\left(e^{-\left(\beta x_{3}+\alpha y_{3}\right)}+e^{-\left(\beta y_{3}+\alpha x_{3}\right)}\right),
\end{aligned}
$$


where $d_{1 / 2}=x_{3} \mp y_{3}$ and

$$
\begin{array}{cc}
R^{ \pm}=\left(\xi^{2}+\beta^{2}\right)^{2} \pm 4 \xi^{2} \alpha \beta, & \alpha=\sqrt{\xi^{2}-k_{p}^{2}}, \quad \beta=\sqrt{\xi^{2}-k_{s}^{2}} \\
k_{p}=\omega \sqrt{\frac{\rho}{\lambda+2 \mu}}, & k_{s}=\omega \sqrt{\frac{\rho}{\mu}} .
\end{array}
$$

One may observe that the kernels (A.3) are characterized by intrinsic symmetries

$$
\begin{array}{rlrl}
\gamma_{1}(\xi ; \boldsymbol{x}, \boldsymbol{y}, \omega) & =\gamma_{1}(\xi ; \boldsymbol{y}, \boldsymbol{x}, \omega), & & \gamma_{2}(\xi ; \boldsymbol{x}, \boldsymbol{y}, \omega)=\gamma_{2}(\xi ; \boldsymbol{y}, \boldsymbol{x}, \omega), \\
\gamma_{3}(\xi ; \boldsymbol{x}, \boldsymbol{y}, \omega)=\Omega_{1}(\xi ; \boldsymbol{y}, \boldsymbol{x}, \omega), & \Omega_{2}(\xi ; \boldsymbol{x}, \boldsymbol{y}, \omega)=\Omega_{2}(\xi ; \boldsymbol{y}, \boldsymbol{x}, \omega),
\end{array}
$$

with respect to the source-receiver arrangement. With the aid of (A.2) and (A.5), it can be directly verified that the displacement Green's functions (A.1) exhibit spatial reciprocity wherein

$$
\hat{u}_{j}^{k}(\boldsymbol{x}, \boldsymbol{y}, \omega)=\hat{u}_{k}^{j}(\boldsymbol{y}, \boldsymbol{x}, \omega), \quad \boldsymbol{x}, \boldsymbol{y} \in \Omega, \quad j, k \in\{1,2,3\} .
$$

